\title{
Black hole perturbations in modified gravity
}

\author{
David Langlois, ${ }^{1}$ Karim Noui, ${ }^{2,1}$ and Hugo Roussille ${ }^{1,2}$ \\ ${ }^{1}$ Université de Paris, CNRS, Astroparticule et Cosmologie, F-75013 Paris, France \\ ${ }^{2}$ Institut Denis Poisson (UMR 7013), Université de Tours, \\ Université d'Orléans, Parc de Grandmont, 37200 Tours, France
}

(Dated: December 15, 2021)

We study the linear perturbations about nonrotating black holes in the context of degenerate higher-order scalar-tensor (DHOST) theories, using a systematic approach that extracts the asymptotic behaviour of perturbations (at spatial infinity and near the horizon) directly from the first-order radial differential system governing these perturbations. For axial (odd-parity) modes, this provides an alternative to the traditional approach based on a second-order Schrödinger-like equation with an effective potential, which we also discuss for completeness. For polar (even-parity) modes, which contain an additional degree of freedom in DHOST theories, and are thus more complex, we use a direct treatment of the four-dimensional first-order differential system (without resorting to a second order reformulation). We illustrate our study with two specific types of black hole solutions: "stealth" Schwarzschild black holes, with a non trivial scalar hair, as well as a class of non-stealth black holes whose metric is distinct from Schwarzschild. The knowledge of the asymptotic behaviours of the perturbations enables us to compute numerically quasi-normal modes, as we show explicitly for the non-stealth solutions. Finally, the asymptotic form of the modes also signals some pathologies in the stealth and non-stealth solutions considered here. 


\section{INTRODUCTION}

The dawn of gravitational wave (GW) astronomy has spurred a renewed interest in possible deviations from General Relativity (GR), which could be detected in the GWs emitted by compact binaries. Of particular interest is the ringdown phase of a binary black hole merger, which can be described by linear perturbations about a background stationary black hole solution. These perturbations mainly correspond to a superposition of quasinormal modes, whose frequencies are quantised (see the reviews [1-4] and references therein). One expects that modified gravity models would predict QNMs that differ from their GR counterpart and the detailed analysis of the GW signal, commonly called "black hole spectroscopy", represents an invaluable window to test General Relativity and to look for specific signatures of modified gravity [5, 6]. So far, QNMs have been investigated only for a few models of modified gravity (see e.g. the review [6] and references therein).

With these motivations in mind, the goal of this paper is to present a new approach for the study of black holes perturbations, illustrated in the context of scalar-tensor theories, which constitute the simplest extensions of Einstein's theory. So far, the most general covariant scalar-tensor theories containing a single scalar degree of freedom are degenerate higher-order scalar-tensor (DHOST) theories, introduced and constructed up to quadratic order (in second derivatives of the scalar field) in [7] and extended up to cubic terms in [8] (see [9] for a review). DHOST theories encompass the traditional scalar-tensor theories (see e.g. [10] and references therein), Horndeski's theories [11] and Beyond Horndeski theories such as the disformal transformations of GR [12] and GLPV theories [13].

There already exists a significant literature on black holes in DHOST theories [14 24 or in subclasses like Horndeski theories (see the review [25] and references therein) and Beyond Horndeski theories [26]. Among the solutions discussed in the literature, one can distinguish the so-called stealth black holes, corresponding to solutions with a non-trivial scalar field profile but with a metric that exactly coincides with a GR black hole solution (possibly with a cosmological constant). These solutions have been scrutinised in detail as they could naturally be compatible with present observations, while leading to specific signatures, at the level of perturbations, that could be detected or constrained by observations. It appears however that stealth solutions seem to suffer from strong coupling issues or instabilities [27 29]. As suggested in [30], one possible cure to the strong coupling problem could be a small detuning of the degeneracy condition.

Other black hole solutions, distinct from GR solutions, have also been constructed. Here we will mainly consider a family of solutions introduced in [26], whose metric is formally analogous to that of Reissner-Nordström black hole but with the square of the electric charge effectively negative, which implies that there is a single horizon. In addition, the scalar field has a non trivial profile in this geometry.

Beyond the construction of exact solutions, the linear perturbations of nonrotating black holes in DHOST theories, or in some subclasses, have been studied in a few papers. For background solutions in Horndeski theories with a purely radially dependent scalar field, the axial perturbations were investigated in [31] and the polar perturbations in [32, in both cases by reducing the quadratic action to keep only the physical degrees of freedom. This analysis was extended in [33, 34] to include a linear time dependence of the background scalar profile, although the stability issue was subsequently revisited in [35]. Black hole perturbations were further discussed in [36 38] in the context of DHOST theories. The perturbations of stealth black holes in some DHOST theories were also investigated in [28] and [29], showing that the equation of motion for the (polar) scalar degree of freedom is characterised by a singular effective metric in some cases, or concluding to the existence of a gradient instability in other cases. The perturbations of the stealth Kerr black hole solutions found in [16] were analysed in [39]. 
Perturbations of nonrotating black holes in Horndeski theories were also studied in [40], but in the restrictive case of a constant background scalar field, which excludes the stealth and non-stealth black holes with a non trivial scalar field profile. In this simple case, axial modes satisfy exactly the same equations as in GR, while the equations of motion for the polar perturbations can be rewritten in a matricial Schrödinger-like system. The latter belongs to the family of generalised second-order Schrödinger-like matricial systems considered in [41] to parametrise small deviations from GR and compute the perturbations of quasi-normal frequencies with respect to their GR values.

In the present work, instead of using a second-order system, which in general is more complex that the ansatz considered in [41] and requires a convoluted calculation (as illustrated in [38] for stealth black holes), we resort here to the new approach that we have presented in a companion paper [42], to be referred to as Paper I. This method analyses directly the first-order differential system in its original form and extracts the asymptotic behaviour of the perturbations. This enables us to identify the asymptotic behaviour of the physical modes and, in particular, to estimate numerically the quasi-normal modes, which are defined by their asymptotic boundary conditions. In this way, we are able to get new insights concerning the perturbations of stealth black holes and to explore for the first time the perturbations of a non-stealth solution introduced in [26].

The structure of the paper is the following. In the next section, we present the quadratic DHOST theories and the black hole solutions considered in the rest of the paper. In section III, following the standard method, we write a general Schrödinger-like equation for axial perturbations, which is then applied to our specific cases of interest. In section IV, we revisit the axial perturbations with our novel approach, obtaining the asymptotic behaviours of the modes and computing numerically the QNMs for the non-stealth solution. We then turn, in section $\mathrm{V}$, to the case of polar modes, for which the standard method is not available. We conclude in section VI. Several appendices have also been added to provide more details on a few technical points.

\section{BLACK HOLES IN DHOST THEORIES}

In this section, we give a brief summary of quadratic DHOST theories, focussing on the subclass Ia (according to the classification of [43]) which contains the most interesting theories from a phenomenological point of view. We then review a few static and spherically symmetric black hole solutions in these theories.

\section{A. Quadratic DHOST theories}

Allowing for second-order derivatives in the action, the most general family of viable scalartensor theories, which contain a single scalar degree of freedom and are free from Ostrogradski instabilities, can be constructed in a systematic way by requiring the degeneracy of the theories [7]. Quadratic DHOST theories are described by an action of the form

$$
S=\int d^{4} x \sqrt{-g}\left[P(X, \phi)+Q(X, \phi) \square \phi+F(X, \phi)^{(4)} R+\sum_{i=1}^{5} A_{i}(X, \phi) L_{i}\right]
$$

where ${ }^{(4)} R$ is the Ricci scalar for the metric $g_{\mu \nu}$ and the $L_{i}$ denote the five possible scalar terms quadratic in second derivatives of $\phi$, namely

$$
\begin{aligned}
& L_{1} \equiv \phi_{\mu \nu} \phi^{\mu \nu}, \quad L_{2} \equiv(\square \phi)^{2}, \quad L_{3} \equiv \phi^{\mu} \phi_{\mu \nu} \phi^{\nu} \square \phi, \\
& L_{4} \equiv \phi^{\mu} \phi_{\mu \nu} \phi^{\nu \rho} \phi_{\rho}, \quad L_{5} \equiv\left(\phi^{\mu} \phi_{\mu \nu} \phi^{\nu}\right)^{2},
\end{aligned}
$$


using the short-hand notations $\phi_{\mu} \equiv \nabla_{\mu} \phi$ and $\phi_{\mu \nu} \equiv \nabla_{\nu} \nabla_{\mu} \phi$ for the first and second (covariant) derivatives of $\phi$. The action contains eight functions, $A_{i}, F, Q$ and $P$, which depend on the scalar field $\phi$ and its kinetic term $X \equiv \phi_{\mu} \phi^{\mu}$. While the functions $P$ and $Q$ are arbitrary, the functions $F$ and $A_{i}$ must satisfy three algebraic conditions [7, in order to ensure the degeneracy of the theory and the absence of any Ostrogradski ghost.

As shown in [43, 44], quadratic DHOST theories can be classified into several classes and subclasses which are stable under general disformal transformations, i.e. transformations of the metric of the form

$$
g_{\mu \nu} \longrightarrow \tilde{g}_{\mu \nu}=C(X, \phi) g_{\mu \nu}+D(X, \phi) \phi_{\mu} \phi_{\nu},
$$

where $C$ and $D$ are arbitrary functions such that the two metrics $g_{\mu \nu}$ and $\tilde{g}_{\mu \nu}$ are not degenerate. Note that, when the disformal transformation is not invertible, one gets mimetic theories of gravity [45, 46], which can also be seen as DHOST theories [47, 48]. As shown recently in [49], invertible disformal transformations can also be used to exhibit a remarkably simple Lagrangian for quadratic DHOST theories when ignoring matter.

Similarly, the theories belonging to class Ia can be mapped into a Horndeski form by applying a disformal transformation. The other classes are not physically viable (either tensor modes have pathological behaviour [50] or gradient instabilities of cosmological perturbations are present [51]) and will not be considered in the present work. Theories in class Ia are specified by the three free functions $F, A_{1}$ and $A_{3}$ (in addition to $P$ and $Q$ ) and the three remaining functions $A_{2}, A_{4}$ and $A_{5}$ are given by algebraic relations in terms of $A_{1}, A_{3}, F$ and $F_{X}$ (which denotes the derivative of $F(X, \phi)$ with respect to $X)$. These relations are a direct consequence of the three degenerate conditions, necessary to guarantee that only one scalar degree of freedom is present [7, 50]. In summary, this means that all the DHOST theories we consider here are characterized by the five functions $P, Q, F, A_{1}$ and $A_{3}$.

Finally, matter can easily be included by adding to the DHOST action an action $S_{m}$ where the matter degrees of freedom are minimally coupled to the metric $g_{\mu \nu}$, which therefore corresponds to the physical metric. Note that this implies that two DHOST theories that are disformally related via (2.3) are physically inequivalent when matter is included (assuming matter minimally coupled to $g_{\mu \nu}$ for the first theory and to $\tilde{g}_{\mu \nu}$ for the second one).

If one is interested only in vacuum solutions, it can be convenient to use these disformal transformations to restrict the study of DHOST Ia theories to their Horndeski subclass, defined by the action

$$
\left.S=\int \mathrm{d}^{4} x \sqrt{-g}\left[F(X, \phi){ }^{(4)} R+P(X, \phi)+Q(X, \phi) \square \phi+2 F_{X}(X, \phi)(\square \phi)^{2}-\phi_{\mu \nu} \phi^{\mu \nu}\right)\right],
$$

i.e. DHOST Ia theories (2.1) with the restrictions

$$
A_{1}=-A_{2}=2 F_{X}, \quad A_{3}=A_{4}=A_{5}=0 .
$$

For simplicity, in the following, we will study nonrotating black holes in gravitational theories described by the above Horndeski action.

\section{B. Black hole solutions}

We now consider static spherically symmetric black hole solutions, i.e. with a metric of the form

$$
g_{\mu \nu} \mathrm{d} x^{\mu} \mathrm{d} x^{\nu}=-A(r) \mathrm{d} t^{2}+\frac{1}{B(r)} \mathrm{d} r^{2}+r^{2}\left(\mathrm{~d} \theta^{2}+\sin ^{2} \theta \mathrm{d} \varphi^{2}\right),
$$


where $A$ and $B$ are functions of the radial coordinate $r$ only. Although it seems natural to assume a radially dependent scalar field, i.e. of the form $\phi=\phi(r)$, it was realised in [52] that one can adopt the more general ansatz

$$
\phi(t, r)=q t+\psi(r),
$$

where $q$ is constant, in the context of shift-symmetric theories, i.e. where the arbitrary functions entering in the DHOST action (2.1) depend only on $X$ and not on $\phi$. In this case, since only the gradient of the scalar field $\phi_{\mu}$ is relevant, 2.7 is compatible with a static metric. Note that if $q \neq 0$ the disformal transformation of the metric (2.6) does not conserve the same form, because of the presence of a nonzero $\tilde{g}_{t r} \neq 0$. This implies that, in the case $q \neq 0$, working with the Horndeski action is more restrictive than starting with the general DHOST action.

Even though our approach is general, in the following we will mainly concentrate on two families of interesting solutions found in the literature, which we now introduce.

\section{Stealth solutions}

Stealth solutions are solutions for which the metric coincides with a vacuum solution of General Relativity, possibly with a cosmological constant. This means that, even if the scalar field profile is non trivial, i.e. $\phi$ non constant, its effective energy-momentum tensor reduces to that of a cosmological constant. These solutions have been actively studied in the context of Horndeski, beyond Horndeski and more generaly DHOST theories in the last few years [14, 15, 17, 19, 25, 26, 36. 53.1 .

For shift-symmetric DHOST Ia theories, or more specifically Horndeski theories, one can obtain stealth Schwarzschild solutions with a scalar field satisfying 2.7) if the conditions

$$
X\left(x^{\mu}\right)=X_{0}=-q^{2}, \quad P\left(X_{0}\right)=P_{X}\left(X_{0}\right)=Q_{X}\left(X_{0}\right)=0,
$$

are satisfied ${ }^{2}$. More concretely, the equations of motion involve the functions $F, P$ and $Q$ up to their second derivatives only evaluated at the background value $X_{0}=-q^{2}$, as can be seen in Appendix B,

As a consequence, if we fix $F\left(X_{0}\right)=1$ for convenience, the only theory-dependent parameters that appear in the equations of motion are

$$
\alpha \equiv F_{X}\left(X_{0}\right), \quad \beta \equiv F_{X X}\left(X_{0}\right), \quad \gamma \equiv P_{X X}\left(X_{0}\right), \quad \delta \equiv Q_{X X}\left(X_{0}\right) .
$$

In other words, without loss of generality, we can limit our study to Horndeski theories with

$$
\begin{aligned}
& F(X) \equiv 1+\alpha\left(X+q^{2}\right)+\frac{\beta}{2}\left(X+q^{2}\right)^{2}, \\
& P(X) \equiv \frac{\gamma}{2}\left(X+q^{2}\right)^{2}, \quad Q(X) \equiv \frac{\delta}{2}\left(X+q^{2}\right)^{2} .
\end{aligned}
$$

All the other terms in the expansions in powers of $\left(X+q^{2}\right)$ of these functions are irrelevant.

The stealth Schwarzschild solution is then described by the metric 2.6) with

$$
A(r)=B(r)=1-\frac{r_{\mathrm{s}}}{r}
$$

\footnotetext{
${ }^{1}$ Note that stealth solutions were first introduced in the context of three-dimensional gravity 54 and an earlier stealth solution in four-dimensional modified gravity was discovered [55] in the context of ghost condensate [56] (even though it was not named "stealth").

${ }^{2}$ More general conditions to get stealth Schwarzschild solutions in DHOST Ia theories are A2 and A3.
} 
where $r_{\mathrm{s}}$ denotes the Schwarzschild radius, and the scalar field 2.7 with ${ }^{3}$

$$
\psi^{\prime}(r)=q \frac{\sqrt{r r_{\mathrm{s}}}}{r-r_{\mathrm{s}}}, \quad \text { (stealth Schwarzschild) }
$$

which is obtained by solving $X=-q^{2}$ (see [52]). Throughout this paper, a prime denotes a derivative with respect to the radial coordinate $r$.

\section{Babichev-Charmousis-Lehébel (BCL) solutions}

While it is natural to look for stealth solutions in alternative theories of gravity, it is more interesting to find genuinely new solutions, i.e. non-stealth solutions. For DHOST theories, this is not an easy task as the equations of motion are quite involved, even for a static and spherically symmetric metric. This is why very few exact non-stealth solutions have been found so far 4 Another approach is to construct solutions numerically (see e.g. [57] for rotating solutions in Horndeski theories with a cubic Galileon and a k-essence term only).

As an illustration, we study in this work the non-stealth solutions obtained in [26] for a subset of Horndeski theories (2.4) characterized by the functions

$$
F(X)=f_{0}+f_{1} \sqrt{X}, \quad P(X)=-p_{1} X, \quad Q(X)=0,
$$

where $f_{0}, f_{1}$ and $p_{1}$ are constants (we take $f_{0}, p_{1}>0$ ) and $X$ is supposed to be positive. For simplicity, we restrict ourselves to the case where the scalar field 2.7 has no time dependence, i.e. $q=0$.

The black hole solution found in [26], which we will name BCL after the authors, is described by a metric of the form (2.6) with

$$
A(r)=B(r)=\left(1-\frac{r_{+}}{r}\right)\left(1+\frac{r_{-}}{r}\right),
$$

where $r_{-}$and $r_{+}$are defined by the relations

$$
r_{+} r_{-}=\frac{f_{1}^{2}}{2 f_{0} p_{1}}, \quad r_{+}-r_{-}=r_{\mathrm{m}} \equiv 2 m, \quad r_{+}>r_{-}>0 .
$$

Note that the expression for $A(r)$ is reminiscent of the Reissner-Nordström metric but with a negative root here. As a consequence, the black hole exhibits a single event horizon, of radius $r_{+}$, in contrast with the Reissner-Nordstroem geometry.

As for the scalar field, its kinetic term is given by

$$
X(r)=A(r) \phi^{\prime 2}(r)=\frac{f_{1}^{2}}{p_{1}^{2} r^{4}},
$$

which is non constant, in contrast with the stealth solutions presented above. The scalar field profile can be found explicitly by integrating the equation

$$
\phi^{\prime}(r)= \pm \frac{f_{1}}{p_{1} r \sqrt{\left(r-r_{+}\right)\left(r+r_{-}\right)}},
$$

\footnotetext{
${ }^{3}$ Note that the equations of motion lead to $\psi^{\prime}$ up to a global sign. Here we make one choice because it gives a regular expression (in Eddington-Finkelstein coordinates) while the expression with the opposite sign leads to a singular scalar field on the horizon [52. However, such a singularity has no physical consequences because $X$ itself and the stress-tensor energy are not singular.

4 A new generic method to construct non-stealth solutions in DHOST theories has been introduced recently in [18. The idea consists in using a known solution $\left(g_{\mu \nu}, \phi\right)$ of a given DHOST theory to build, via a disformal transformation (2.3), a new solution $\left(\tilde{g}_{\mu \nu}, \phi\right)$ for the disformally related DHOST theory. In general, a stealth solution transforms into a non-stealth one. An interesting result from this method is the construction of the first non-stealth rotating black hole solutions in DHOST theories 20, 21.
} 
yielding $9^{5}$

$$
\phi(r)= \pm \frac{f_{1}}{p_{1} \sqrt{r_{+} r_{-}}} \arctan \left[\frac{r_{\mathrm{m}} r+2 r_{+} r_{-}}{2 \sqrt{r_{+} r_{-}} \sqrt{\left(r-r_{+}\right)\left(r+r_{-}\right)}}\right]+\text {cst } .
$$

This concludes our presentation of the background solutions, whose perturbations will be considered in the following.

\section{AXIAL PERTURBATIONS: STANDARD APPROACH}

The rest of this paper is devoted to the study of the dynamics of linear perturbations about the black hole solutions described in the previous section. In this section and the next one, we examine the axial (or odd-parity) perturbations, which are simpler to analyse than polar (or even-parity) perturbations discussed in section $\mathrm{V}$. Axial perturbations correspond to the perturbations of the metric that transform like $(-1)^{\ell}$ under parity transformation, when decomposed into spherical harmonics, where $\ell$ is the usual multipole integer.

In this section, we follow the standard approach for black hole perturbations which consists in reformulating the linearised equations of motion as a second order Schrödinger-like equation. In particular, we derive the corresponding effective potential for both stealth Schwarzschild and BCL black hole solutions.

\section{A. Equations of motion for the perturbations}

To derive the linearised equations of motion, let us substitute the perturbed metric and scalar field,

$$
g_{\mu \nu}=\bar{g}_{\mu \nu}+h_{\mu \nu}, \quad \phi=\bar{\phi}+\delta \phi,
$$

where a bar denotes a background quantity, into the gravitational scalar-tensor action (2.1), or (2.4), and expand it up to second order in the perturbations $h_{\mu \nu}$ and $\delta \phi$. The quadratic part of the action, $S_{\text {quad }}\left[h_{\mu \nu}, \delta \phi\right]$ then describes the dynamics of linear perturbations and the linearised equations of motion are given by the associated Euler-Lagrange equations,

$$
\mathcal{E}_{\mu \nu} \equiv \frac{\delta S_{\text {quad }}}{\delta h_{\mu \nu}}=0, \quad \mathcal{E}_{\phi} \equiv \frac{\delta S_{\text {quad }}}{\delta \phi}=0 .
$$

The equation $\mathcal{E}_{\phi}=0$ turns out to be redundant as a consequence of Bianchi's identities, so we just need to take into account the metric equations $\mathcal{E}_{\mu \nu}=0$.

We now assume a background metric $\bar{g}_{\mu \nu}$ of the form (2.6), keeping $A(r)$ and $B(r)$ unspecified at this stage, and a scalar field (2.7). In terms of the spherical harmonics $Y_{\ell m}$ and working in the traditional Regge-Wheeler gauge (details about gauge fixing can be found in paper I), the axial metric perturbations for $\ell \geq 2$ read explicitly

$$
\begin{array}{ll}
h_{t \theta}=\frac{1}{\sin \theta} \sum_{\ell, m} h_{0}^{\ell m}(t, r) \partial_{\varphi} Y_{\ell m}(\theta, \varphi), & h_{t \varphi}=-\sin \theta \sum_{\ell, m} h_{0}^{\ell m}(t, r) \partial_{\theta} Y_{\ell m}(\theta, \varphi), \\
h_{r \theta}=\frac{1}{\sin \theta} \sum_{\ell, m} h_{1}^{\ell m}(t, r) \partial_{\varphi} Y_{\ell m}(\theta, \varphi), & h_{r \varphi}=-\sin \theta \sum_{\ell, m} h_{1}^{\ell m}(t, r) \partial_{\theta} Y_{\ell m}(\theta, \varphi),
\end{array}
$$

\footnotetext{
${ }^{5}$ The sign of $\phi(r)$ and the constant are physically irrelevant. Notice that the derivative of the scalar field diverges at the horizon. According to [26, this is not a problem as it is a coordinate dependent statement which disappears in the tortoise coordinate for instance. Furthermore, it was argued in 26] that all physical meaningful quantities are well-defined at the horizon, for e.g. the scalar field itself.

${ }^{6}$ As in GR, the dipole perturbation $(\ell=1)$ does not not propagate 36].
} 
while all the other components vanish. Moreover, the scalar field perturbation is zero by construction for axial modes. All the modes $(\ell m)$ decouple at the linear level and, in the following, we will drop this label to shorten the notation.

Since the metric is static, it is also convenient to decompose any time-dependent function $f(t, r)$ in Fourier modes, according to

$$
f(t, r)=\int_{-\infty}^{+\infty} \mathrm{d} \omega f(\omega, r) \exp (-i \omega t)
$$

In practice, this implies that all partial derivatives with respect to time become, in Fourier space, multiplications by $-i \omega$. The equations of motion for the Fourier modes, which we will also denote $\mathcal{E}_{\mu \nu}=0$, therefore consist of a system of ordinary differential equations, with only derivatives with respect to the variable $r$. As discussed in Appendix C1, the only relevant equations of motion reduce to

$$
\mathcal{E}_{r \theta}=0, \quad \mathcal{E}_{\theta \theta}=0
$$

These two equations are first order ordinary differential equations and, after using the background equations of motion (see Appendix B), they drastically simplify into a differential system for the two functions

$$
Y_{1}(r)=h_{0}(r), \quad Y_{2}(r) \equiv \frac{1}{\omega}\left(h_{1}+\Psi h_{0}\right),
$$

which reads, using $Y={ }^{T}\left(Y_{1}, Y_{2}\right)$,

$$
\frac{\mathrm{d} Y}{\mathrm{~d} r}=M(r) Y, \quad M \equiv\left(\begin{array}{cc}
2 / r+i \omega \Psi & -i \omega^{2}+2 i \lambda \Phi / r^{2} \\
-i \Gamma & \Delta+i \omega \Psi
\end{array}\right)
$$

where

$$
\lambda \equiv \frac{(\ell-1)(\ell+2)}{2}
$$

and we have introduced the four functions

$$
\begin{aligned}
\Psi & \equiv \frac{2 q F_{X} \psi^{\prime}}{\mathcal{F}}, \quad \Phi \equiv \frac{\mathcal{F}}{F-2 X F_{X}}, \quad \Gamma \equiv \frac{A F\left(F-2 X F_{X}\right)}{B \mathcal{F}^{2}} \\
\Delta & \equiv \frac{\sqrt{A / B}}{\mathcal{F}}\left[A B\left(\frac{F}{\sqrt{A B}}\right)^{\prime}+2 X\left(\sqrt{A B} F_{X}\right)^{\prime}+2 q^{2}\left(\sqrt{\frac{B}{A}} F_{X}\right)^{\prime}\right],
\end{aligned}
$$

which depend on the function $\mathcal{F}$ defined by

$$
\mathcal{F} \equiv A\left(F-2 X F_{X}\right)-2 q^{2} F_{X}
$$

In General Relativity, the functions defined above reduce to

$$
\Psi=0, \quad \Phi=A, \quad \Gamma=A^{-2}, \quad \Delta=-A^{\prime} / A,
$$

with $\mathcal{F}=A$, and 3.7 reduces to the system studied in Paper I. 


\section{B. Schrödinger-like equation and effective potential}

Following the standard approach (originally introduced in [58] and recalled in Paper I), we now recast the above system (3.7) into a single Schrödinger-like equation, which is second order with respect to the radial coordinate $r$ and depends on $\omega^{2}$ (corresponding to a second order time derivative).

As discussed in detail in Appendix D, a transformation

$$
Y(r)=\hat{P}(r) \hat{Y}(r)
$$

with the appropriate matrix $]^{7} \hat{P}$ enables us to rewrite the system (3.7) in the canonical form

$$
\frac{\mathrm{d} \hat{Y}}{\mathrm{~d} r_{*}}=\left(\begin{array}{cc}
i \mu(r) \omega & 1 \\
V(r)-\omega^{2} / c^{2}(r) & i \mu(r) \omega
\end{array}\right) \hat{Y}
$$

where we have introduced a new radial coordinate $r_{*}$ and the functions $\mu$ and $c$, defined by

$$
\frac{\mathrm{d} r}{\mathrm{~d} r_{*}} \equiv n, \quad \mu \equiv n \Psi, \quad c^{2} \equiv \frac{1}{n^{2} \Gamma} .
$$

In terms of the functions introduced in (3.9)- (3.10), the potential $V$ in (3.14) reads (see Appendix $\mathrm{D}$ for the explicit calculation)

$$
\begin{gathered}
V=\frac{n^{2}}{4}\left[\frac{8(1+\lambda \Phi \Gamma)}{r^{2}}+\Delta^{2}-\frac{4 \Delta}{r}+2 \Delta^{\prime}+\frac{2 \Gamma^{\prime}}{\Gamma}\left(\frac{2}{r}-\Delta\right)\right. \\
\left.+3\left(\frac{\Gamma^{\prime}}{\Gamma}\right)^{2}+\left(\frac{n^{\prime}}{n}\right)^{2}-2\left(\frac{\Gamma^{\prime \prime}}{\Gamma}+\frac{n^{\prime \prime}}{n}\right)\right] .
\end{gathered}
$$

One can check that this coincides with the expression found in 36$]^{8}$ in the case $n=\sqrt{A B}$. Let us stress that the explicit expressions of propagation speed $c(r)$ and of the potential $V(r)$ depend on the choice of the radial coordinate $r_{*}$, characterised by $n$.

In contrast to General Relativity, where $\Psi=0$, one cannot eliminate in general the diagonal components in the new matrix above via a change of functions (3.13). However, as noticed in [36], this can be achieved via a time redefinition of the form?

$$
t \longrightarrow t+\nu(r) \text {. }
$$

Indeed, under such a time coordinate change, one easily show that the system (3.14) transforms into

$$
\frac{\mathrm{d} \hat{Y}}{\mathrm{~d} r_{*}}=\left(\begin{array}{cc}
i \omega\left(\mu(r)+\mathrm{d} \nu / \mathrm{d} r_{*}\right) & 1 \\
V(r)-\omega^{2} / c^{2}(r) & i \omega\left(\mu(r)+\mathrm{d} \nu / \mathrm{d} r_{*}\right)
\end{array}\right) \hat{Y}
$$

We can then eliminate the diagonal terms by choosing

$$
\nu(r)=-\int \frac{\mu(r)}{n(r)} \mathrm{d} r=-\int \Psi(r) \mathrm{d} r
$$

This choice of change of time variable leads to a Schrödinger-like equation, of the form

$$
\frac{\mathrm{d}^{2} \hat{Y}_{1}}{\mathrm{~d} r_{*}^{2}}+\left(\frac{\omega^{2}}{c^{2}(r)}-V(r)\right) \hat{Y}_{1}=0
$$

where $c(r)$ corresponds to the propagation speed and $V(r)$ to the effective potential.

\footnotetext{
7 The matrix $\hat{P}$ here corresponds to the matrix $\tilde{P} \hat{P}$ in Appendix Dhere $\tilde{P}$ is defined in $[\mathrm{D} 18]$ and $\hat{P}$ in D8 .

${ }^{8}$ See Eq. (64) of [36]. We do not recover, however, the potential used in [59] in the limit $q \longrightarrow 0$. An unfortunate consequence is that the computation of quasi-normal modes in 60. should be revisited as the latter potential was used in that work.

${ }^{9}$ Equivalently, one can get rid of the diagonal terms by a redefinition $\hat{Y} \longrightarrow e^{-i \omega \nu(r)} \hat{Y}$, where $\nu(r)$ is given by 3.19 .
} 


\section{Stealth Schwarzschild axial pertubations}

Let us apply the above results to the stealth Schwarzschild solution described in section IIB 1. Substituting the background expressions (2.11) and (2.12) into (3.9) and (3.10), one finds

$$
\begin{aligned}
& \Psi=\frac{\zeta r_{\mathrm{s}}^{1 / 2} r^{3 / 2}}{\left(r-r_{\mathrm{s}}\right)\left(r-r_{g}\right)}, \quad \Phi=\frac{r-r_{g}}{(1+\zeta) r}, \\
& \Gamma=\frac{(1+\zeta) r^{2}}{\left(r-r_{g}\right)^{2}}, \quad \Delta=\frac{1}{r}-\frac{1}{r-r_{g}},
\end{aligned}
$$

where we have introduced the constant parameters

$$
\zeta \equiv 2 q^{2} \alpha \geq 0, \quad r_{g} \equiv(1+\zeta) r_{\mathrm{s}} .
$$

This dimensionless constant $\zeta$ parametrises the deviation from General Relativity, since one recovers the GR functions (3.12) when $\zeta=0$. The radius $r_{g}$, which differs from $r_{\mathrm{s}}$ when $\zeta \neq 0$, appears as an extra pole in the above functions, in addition to $r_{\mathrm{s}}$ and 0 .

From the expressions (3.21), one can compute the potential $V(r)$ and the propagation speed $c(r)$ that appear in the Schrödinger-like equation (3.20). As already stressed in the previous subsection, these quantities depend on the choice of the radial coordinate. If one adopts the usual Schwarzschild tortoise coordinate, defined by

$$
r_{*}=\int \mathrm{d} r \frac{r}{r-r_{\mathrm{s}}}=r+r_{\mathrm{s}} \ln \left(r / r_{\mathrm{s}}-1\right),
$$

corresponding to the choice $n=A(r)=1-r_{\mathrm{s}} / r$, the potential takes the form

$$
V(r)=\frac{V_{0}+V_{1}\left(r_{\mathrm{s}} / r\right)+V_{2}\left(r_{\mathrm{s}} / r\right)^{2}+V_{3}\left(r_{\mathrm{s}} / r\right)^{3}+V_{4}\left(r_{\mathrm{s}} / r\right)^{4}}{\left(r-r_{g}\right)^{2}},
$$

with

$$
\begin{aligned}
& V_{0}=2(\lambda+1), \quad V_{1}=-2(\lambda+3) \zeta-6 \lambda-9, \quad V_{2}=(15 \zeta+16 \lambda+70) \zeta / 4+6 \lambda+15, \\
& V_{3}=-(1+\zeta)(13 \zeta / 2+2 \lambda+11), \quad V_{4}=3(1+\zeta)^{2},
\end{aligned}
$$

and the propagation speed is given by the expression

$$
c(r)=\frac{r-r_{g}}{\sqrt{1+\zeta}\left(r-r_{\mathrm{s}}\right)},
$$

where one must take $\zeta>-1$ in order to have $c^{2}>0$.

Another possibility is to choose the radial coordinate such that the propagation speed is $c=1$, i.e.

$$
r_{*}=\int \mathrm{d} r \sqrt{\Gamma}=\sqrt{1+\zeta}\left[r+r_{g} \ln \left(r / r_{g}-1\right)\right],
$$

which is very similar to the usual tortoise coordinate, with $r_{g}$ instead of $r_{\mathrm{s}}$ and a global rescaling. In this case, the potential becomes

$$
V_{c=1}(r)=\left(1-\frac{r_{g}}{r}\right) \frac{2(\lambda+1) r-3 r_{g}}{(1+\zeta) r^{3}},
$$

which is, quite remarkably, identical to the standard Regge-Wheeler potential, with $r_{g}$ instead of $r_{\mathrm{s}}$, up to a global rescaling. One can note that $r_{\mathrm{s}}$ has completely disappeared from the equation of 
motion and $r_{g}$ seems to play the role of the horizon that is effectively "seen" by the axial metric perturbations. The same result was obtained recently in [37] by analysing the effective metric that appears in the equation of motion for the axial perturbations.

In fact, this result can be understood by noting that the quadratic Lagrangian for the axial tensor perturbations can come only from two terms in $(2.1)$ : the term in ${ }^{(4)} R$, which contains both $K_{\mu \nu} K^{\mu \nu}$ and ${ }^{(3)} R$ according to the Gauss-Codazzi identity ${ }^{10}$, and the Lagrangian $L_{1}$ in $(2.2)$, which contains $K_{\mu \nu} K^{\mu \nu}$ (see discussion in [61]). These two terms give, in the quadratic Lagrangian of the axial modes, a kinetic term with coefficient $F-X A_{1}$, evaluated on the background, and a gradient terms with coefficient given by $F$, again evaluated on the background. Since $X$ is a constant, these coefficients are constant and, when $A_{1}=0$, one recovers the same quadratic Lagrangian as in GR with $c=1$. Even if $A_{1} \neq 0$ (which is the case here since $A_{1}=2 F_{X}=2 \alpha$ ), it is possible to perform a disformal transformation to go into a "frame" where $A_{1}=0$ and therefore $c=1$. The background metric is disformally transformed into a new metric, which is straightforward to compute using the disformal transformations of quadratic DHOST theories given in [43]. It turns out that this new metric is another stealth Schwarzschild metric with a displaced horizon, corresponding to $r_{g}$, as discussed in [18], which explains why the potential in this frame coincides with the standard Regge-Wheeler potential.

\section{BCL axial pertubations}

We now apply the results of section III B to the non-stealth solution described in section IIB 2 . In this case, the new coordinate $r_{*}$ is given by

$$
r_{*}=\int \mathrm{d} r \frac{r^{2}}{\left(r-r_{+}\right)\left(r+r_{-}\right)}=r+\frac{r_{+}^{2} \ln \left(r / r_{+}-1\right)-r_{-}^{2} \ln \left(r / r_{-}+1\right)}{r_{+}+r_{-}} .
$$

For the BCL background, characterised by 2.14 with 2.15$)$, we find that the functions 3.9 and (3.10) entering in the coefficients of the differential system (3.7) read

$$
\begin{aligned}
& \Psi=0, \quad \Phi=A, \quad \Gamma=\frac{F}{f_{0} A^{2}}=\frac{r^{2}\left(r^{2}+2 r_{+} r_{-}\right)}{\left(r-r_{+}\right)^{2}\left(r+r_{-}\right)^{2}}, \\
& \Delta=-\frac{A^{\prime}}{A}=-\frac{r_{+}}{r\left(r-r_{+}\right)}+\frac{r_{-}}{r\left(r+r_{-}\right)},
\end{aligned}
$$

since $\mathcal{F}=f_{0} A$.

Furthermore, the potential (3.16) takes the form

$$
V(r)=A(r) \frac{V_{0}+V_{1}\left(r_{\mathrm{m}} / r\right)+V_{2}\left(r_{\mathrm{m}} / r\right)^{2}+V_{4}\left(r_{\mathrm{m}} / r\right)^{4}+V_{6}\left(r_{\mathrm{m}} / r\right)^{6}}{2 r^{2}\left(1+\xi\left(r_{\mathrm{m}} / r\right)^{2}\right)^{2}},
$$

with the coefficients

$$
V_{0}=4(\lambda+1), \quad V_{1}=-6, \quad V_{2}=6(2 \lambda-1) \xi, \quad V_{4}=(12 \lambda-1) \xi^{2}, \quad V_{6}=4 \lambda \xi^{3},
$$

and where we have introduced the dimensionless constant

$$
\xi \equiv 2 \frac{r_{+} r_{-}}{r_{\mathrm{m}}^{2}}=\frac{f_{1}^{2}}{f_{0} p_{1} r_{\mathrm{m}}^{2}}
$$

${ }^{10}$ In a $(3+1)$ decomposition of spacetime, where $n^{\mu}$ is the unit vector normal to the spatial hypersurfaces, the Gauss-Codazzi equation reads ${ }^{(4)} R=K_{\mu \nu} K^{\mu \nu}-K^{2}+{ }^{(3)} R+2 \nabla_{\mu}\left(K n^{\mu}-n^{\nu} \nabla_{\nu} n^{\mu}\right)$, where $h_{\mu \nu} \equiv g_{\mu \nu}+n_{\mu} n_{\nu}$ and $K_{\mu \nu} \equiv h_{\mu}^{\alpha} \nabla_{\alpha} n_{\nu}$. 
Similarly to the parameter $\zeta$ in the stealth case, $\xi$ parametrises the deviation from GR (corresponding to the limit $r_{-}=0$, i.e. $\left.f_{1}=0\right)$.

One notes that one must have $\xi \geq 0$ to prevent a singularity in the potential. When $\xi=0$, one recovers the standard Regge-Wheeler (RW) potential for the Schwarzschild geometry,

$$
V_{\mathrm{RW}}(r)=\left(1-\frac{r_{\mathrm{s}}}{r}\right) \frac{2(\lambda+1) r-3 r_{\mathrm{s}}}{r^{3}},
$$

where $r_{\mathrm{s}}=r_{\mathrm{m}}$ in this limit. Potentials for several values of $\xi$ are shown on Fig. 1, where one can see that the potential is a deformation, parametrised by $\xi$, of the RW potential. At infinity, the behaviour of the potential is very similar to that of the RW potential, with corrections appearing only at second order in $r_{\mathrm{m}} / r$ :

$$
V(r)=\frac{1}{r_{\mathrm{m}}^{2}}\left[2(\lambda+1) \frac{r_{\mathrm{m}}^{2}}{r^{2}}-(2 \lambda+5) \frac{r_{\mathrm{m}}^{3}}{r^{3}}+\mathcal{O}\left(\frac{r_{\mathrm{m}}^{4}}{r^{4}}\right)\right]
$$

By contrast, the leading order behaviour is modified near the horizon,

$$
V(r)=\frac{32 \mu_{\xi}\left(\lambda\left(3 \mu_{\xi}-1\right)^{2}-\mu_{\xi}\left(1+\mu_{\xi}\right)\right)}{\left(1+\mu_{\xi}\right)^{5}\left(3 \mu_{\xi}-1\right) r_{\mathrm{m}}^{3}}\left(r-r_{+}\right)+\mathcal{O}\left(\left(r-r_{+}\right)^{2}\right), \quad \mu_{\xi} \equiv \sqrt{1+2 \xi},
$$

where we have used $r_{ \pm}=r_{\mathrm{m}}\left(1 \pm \mu_{\xi}\right) / 2$. Notice that the height of the potential also depends on the value of $\xi$.

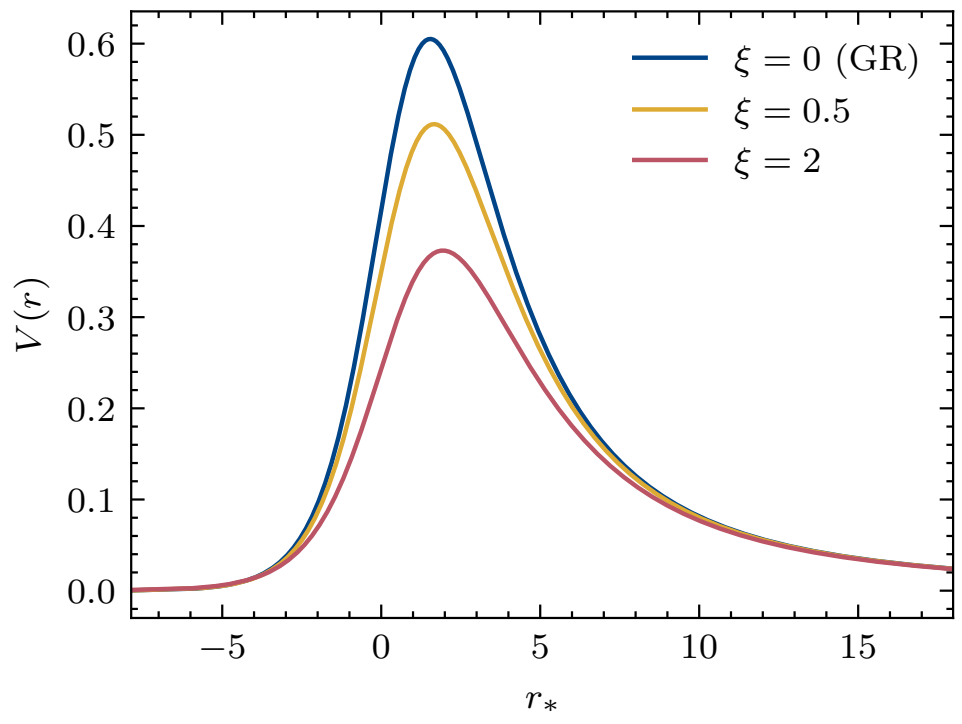

FIG. 1 Potential $V(r)$ for the non-stealth for different values of $\xi$ but fixed values of $r_{\mathrm{m}}=1$ and $\ell=2(\lambda=2)$.

The propagation speed is given by

$$
c(r)=\frac{r}{\sqrt{r^{2}+\xi r_{\mathrm{m}}^{2}}} .
$$

We thus recover the usual value $c=1$ at spatial infinity (when $r \rightarrow \infty$ ), but at the horizon we find

$$
c\left(r_{+}\right)=\sqrt{\frac{r_{+}}{r_{+}+2 r_{-}}}=\sqrt{\frac{\mu_{\xi}+1}{3 \mu_{\xi}-1}} \leq 1 .
$$


From the Schrödinger equation (3.20) with $(3.31)$ and $(3.37)$, one can compute explicitly the complex frequency of the associated quasi-norrmal models (QNM) by resorting to standard numerical techniques [1,3] which were applied in the context of Horndeski theories in [62. In the present case, we will postpone the computation of the QNMs modes to the next section, where we will show that they can be computed numerically even without the Schrödinger-like reformulation of the equations of motion.

\section{AXIAL PERTURBATIONS: FIRST-ORDER SYSTEM APPROACH}

In this section, we revisit axial perturbations and study their asymptotic behaviour, both at infinity and near the horizon, using the method presented in Paper I. Using these asymptotic behaviours, we then compute the quasi-nomal modes numerically.

\section{A. First order approach: method and goal}

Ignoring the traditional Schrödinger reformulation of the perturbation equations, discussed in the previous section, we now go back to the original first-order system and apply the systematic method developed in Paper I to determine the asymptotic behaviour of the solution at spatial infinity and near the black hole horizon.

More precisely, let us consider some first order system of the form

$$
\frac{\mathrm{d} Y}{\mathrm{~d} z}=M(z) Y, \quad M(z)=z^{r} \sum_{n=0}^{p} M_{r-n} z^{-n}+\mathcal{O}\left(z^{r-p-1}\right),
$$

where $Y$ is a column vector, $M(z)$ a square matrix which can expanded, up to some given order, when the variable $z$ goes to infinity. In most cases ${ }^{11}$, the solution $Y(z)$ can be written asymptotically in the form

$$
Y(z)=e^{\Upsilon(z)} z^{\boldsymbol{\Delta}} \mathbf{F}(z) Y_{0}, \quad(z \rightarrow \infty)
$$

where $Y_{0}$ is a constant vector (which can be constrained by boundary conditions), $\mathbf{F}(z)$ is a matrix regular at infinity, $\boldsymbol{\Delta}$ is a constant diagonal matrix, and finally $\mathbf{\Upsilon}(z)$ is also a diagonal matrix whose coefficients are polynomials of degree (at most) $r$. The algorithm described in Paper I, based on [63 66], enables one to compute explicitly all the quantities entering in (4.2), up to some order.

Note that there is no loss of generality when considering the asymptotic behaviour at infinity since one can always reformulate a system that is singular for some finite value $z_{0}$ into a system of the form (4.1) via a change of variable.

In the following, we apply the algorithm of paper I successively to the BCL perturbations and to the stealth Schwarzschild perturbations.

\section{B. BCL axial perturbations}

As found in the previous section, the axial perturbations of the BCL black hole satisfy the system (see (3.7)

$$
\frac{\mathrm{d} Y}{\mathrm{~d} r}=M Y, \quad M(r)=\left(\begin{array}{cc}
2 / r & -i \omega^{2}+i 2 \lambda A / r^{2} \\
-i \Gamma & \Delta
\end{array}\right)
$$

11 The variable $z$ that appears in the asymptotic solution 4.2 can sometimes differ from the original variable in the system (4.1). Moreover, in the very particular cases where the system is such that $M(z)=M_{-1} / z+\mathcal{O}\left(1 / z^{2}\right)$ with $M_{-1}$ nilpotent, the asymptotic expansion of $Y(z)$ is no longer given by 4.2 but it can be expressed as a polynomial of $\ln z$ (see section IV.C of Paper I). 
with, according to 3.30 ,

$$
A=\left(1-\frac{r_{+}}{r}\right)\left(1+\frac{r_{-}}{r}\right), \quad \Gamma=\frac{r^{2}\left(r^{2}+2 r_{+} r_{-}\right)}{\left(r-r_{+}\right)^{2}\left(r+r_{-}\right)^{2}}, \quad \Delta=-\frac{r_{+}}{r\left(r-r_{+}\right)}+\frac{r_{-}}{r\left(r+r_{-}\right)} .
$$

\section{At spatial infinity}

When $r \rightarrow \infty$, the asympotic expansion of the matrix $M(r)$ in 4.3 reads

$$
M(r)=M_{0}+\frac{1}{r} M_{-1}+\mathcal{O}\left(\frac{1}{r^{2}}\right), \quad M_{0} \equiv-i\left(\begin{array}{cc}
0 & \omega^{2} \\
1 & 0
\end{array}\right), \quad M_{-1} \equiv 2\left(\begin{array}{cc}
1 & 0 \\
-i r_{\mathrm{s}} & 0
\end{array}\right),
$$

where we have stopped at order $1 / r$, which will be sufficient for our purpose. Note that the two terms in the above expansion do not depend on $\xi$, which mean they coincide with the analogous terms in GR. This is consistent with the observation that the asymptotic behaviour of the potential (3.31) at infinity coincides with that of the RW potential (3.34) up to first order in $1 / r$.

Since we have already analysed the same asymptotic system in Paper I for the axial modes in Schwarzschild, we recall briefly the main result. Using the transformation

$$
Y=\tilde{P} \tilde{Y}, \quad \tilde{P}=\left(\begin{array}{cc}
-1+\varpi_{+} & 1+\varpi_{-} \\
1+\varpi_{+} & 1-\varpi_{-}
\end{array}\right), \quad \varpi_{ \pm} \equiv \frac{ \pm \omega r_{\mathrm{m}}+i}{2 \omega r},
$$

we obtain the equivalent, and fully diagonalised, system

$$
\frac{\mathrm{d} \tilde{Y}}{\mathrm{~d} r}=\tilde{M} \tilde{Y}, \quad \tilde{M}(r)=\left(\begin{array}{cc}
-i \omega & 0 \\
0 & i \omega
\end{array}\right)+\frac{1}{r}\left(\begin{array}{cc}
1-i \omega r_{\mathrm{m}} & 0 \\
0 & 1+i \omega r_{\mathrm{m}}
\end{array}\right)+\mathcal{O}\left(\frac{1}{r^{2}}\right) .
$$

Direct integration yields the asymptotic solution

$$
\tilde{Y}(r)=(1+\mathcal{O}(1 / r))\left(\begin{array}{l}
a_{-} e^{-i \omega r} r^{1-i \omega r_{\mathrm{m}}} \\
a_{+} e^{+i \omega r} r^{1+i \omega r_{\mathrm{m}}}
\end{array}\right)=(r+\mathcal{O}(1))\left(\begin{array}{c}
a_{-} e^{-i \omega r_{*}} \\
a_{+} e^{+i \omega r_{*}}
\end{array}\right)
$$

where $a_{ \pm}$are arbitrary constants and we have reintroduced, in the last expression, the variable $r_{*}$ associated with the BCL solution, defined in 3.29$)^{12}$

Taking into account the time dependence $e^{-i \omega t}$ of the modes, the two components $\tilde{Y}_{-}$(up component) and $\tilde{Y}_{+}$(down component) of $\tilde{Y}$ take the form

$$
e^{-i \omega t} \tilde{Y}_{\mp}(r)=a_{\mp}(r+\mathcal{O}(1)) e^{-i \omega\left(t \pm r_{*}\right)}
$$

where one recognises the usual ingoing mode (associated with $a_{-}$) and outgoing mode (associated with $\left.a_{+}\right)$at spatial infinity. The values of $a_{ \pm}$can be restricted by the boundary conditions imposed on the system. For example, requiring that the mode is purely outgoing, as is the case for QNMs, imposes $a_{-}=0$.

12 The tortoise coordinate associated with the BCL solution has been computed in 3.29 and its large $r$ expansion reads

$$
r_{*}=r+r_{\mathrm{m}} \ln r-\frac{r_{+}^{2} \ln r_{+}-r_{-}^{2} \ln r_{-}}{r_{+}+r_{-}}-\frac{r_{+}^{2}+r_{-}^{2}-r_{+} r_{-}}{r}+\mathcal{O}\left(\frac{1}{r^{2}}\right) .
$$

When $r_{\mathrm{m}}=r_{\mathrm{s}}=2 m$, it coincides with the Schwarzschild tortoise coordinate $3.23 r_{*}=r+r_{\mathrm{s}} \ln r$ up to the order $\mathcal{O}(1)$. Hence, one can equivalently use any of the two coordinates in the asymptotic 4.8] which has been given up to $\mathcal{O}(1)$ as well. 


\section{At the horizon}

We now turn to the asymptotic behaviour at the black hole horizon. Introducing the variable

$$
\varepsilon \equiv r-r_{+}
$$

the near-horizon asymptotic expansions of the functions $A, \Gamma$ and $\Delta$ in (4.4), are given by

$$
A=\mathcal{O}(\varepsilon), \quad \Gamma=i\left(\frac{\Gamma_{2}}{\varepsilon^{2}}+\frac{\Gamma_{1}}{\varepsilon}+\Gamma_{0}\right)+\mathcal{O}(\varepsilon), \quad \Delta=\frac{\Delta_{1}}{\varepsilon}+\Delta_{0}+\mathcal{O}(\varepsilon) .
$$

Substituting into 4.3), we obtain the asymptotic expansion of the matrix $M$,

$$
M(\varepsilon)=\frac{1}{\varepsilon^{2}}\left(\begin{array}{cc}
0 & 0 \\
\Gamma_{2} & 0
\end{array}\right)+\frac{1}{\varepsilon}\left(\begin{array}{cc}
0 & 0 \\
\Gamma_{1} & \Delta_{1}
\end{array}\right)+\left(\begin{array}{cc}
2 / r_{+} & -i \omega^{2} \\
\Gamma_{0} & \Delta_{0}
\end{array}\right)+\mathcal{O}(\varepsilon),
$$

where we will need only the explicit expression of the coefficients $\Delta_{1}$ and $\Gamma_{2}$,

$$
\Delta_{1}=-1, \quad \Gamma_{2}=-i r_{0}^{2} \quad \text { with } \quad r_{0} \equiv r_{+} \frac{\sqrt{r_{+}\left(r_{+}+2 r_{-}\right)}}{r_{+}+r_{-}} .
$$

Our system now differs from the GR analog studied in Paper I. However, the leading order term is still nilpotent, as in GR, and the resolution of the system is very similar to the analysis of Paper I. According to the algorithm, one first needs to perform the transformation

$$
Y \equiv P_{(1)} Y^{(1)}, \quad \text { with } \quad P_{(1)}(\varepsilon) \equiv\left(\begin{array}{cc}
1 & 0 \\
0 & 1 / \varepsilon
\end{array}\right)
$$

which leads to the new system

$$
\frac{\mathrm{d} Y^{(1)}}{\mathrm{d} \varepsilon}=M^{(1)} Y^{(1)}, \quad M^{(1)}=\frac{1}{\varepsilon}\left(\begin{array}{cc}
0 & -i \omega^{2} \\
\Gamma_{2} & 1+\Delta_{1}
\end{array}\right)+\mathcal{O}(1)=-\frac{i}{\varepsilon}\left(\begin{array}{cc}
0 & \omega^{2} \\
r_{0}^{2} & 0
\end{array}\right)+\mathcal{O}(1) .
$$

The leading term of the new matrix $M^{(1)}$ is now diagonalisable and the system can be explicitly diagonalised via the transformation

$$
Y^{(1)} \equiv P_{(2)} Y^{(2)}, \quad \text { with } \quad P_{(2)}=\left(\begin{array}{cc}
\omega & -\omega \\
r_{0} & r_{0}
\end{array}\right)
$$

leading to the new system

$$
\frac{\mathrm{d} Y^{(2)}}{\mathrm{d} \varepsilon}=M^{(2)} Y^{(2)}, \quad M^{(2)}(\varepsilon)=\frac{i \omega r_{0}}{\varepsilon}\left(\begin{array}{cc}
-1 & 0 \\
0 & 1
\end{array}\right)+\mathcal{O}(1) .
$$

Finally, integrating this system yields

$$
Y^{(2)}(\varepsilon)=(1+\mathcal{O}(\varepsilon))\left(\begin{array}{l}
a_{-} \varepsilon^{-i \omega r_{0}} \\
a_{+} \varepsilon^{+i \omega r_{0}}
\end{array}\right)=(1+\mathcal{O}(\varepsilon))\left(\begin{array}{l}
a_{-} e^{-i \eta \omega r_{*}} \\
a_{+} e^{+i \eta \omega r_{*}}
\end{array}\right),
$$

where $a_{\mp}$ are constants and we have used the asymptotic expansion of the tortoise coordinate 3.29 near the horizon,

$$
r_{*}=\frac{r_{+}^{2}}{r_{+}+r_{-}} \ln \varepsilon+\mathcal{O}(1)=\frac{r_{0}}{\eta} \ln \varepsilon+\mathcal{O}(1), \quad \eta \equiv \frac{\sqrt{r_{+}+2 r_{-}}}{r_{+}^{1 / 2}} .
$$

Taking into account the time dependence $e^{-i \omega t}$, one thus gets for the two components of $Y^{(2)}$

$$
e^{-i \omega t} Y_{\mp}^{(2)}=a_{\mp} e^{-i \omega\left(t \pm \eta r_{*}\right)}(1+\mathcal{O}(\varepsilon)),
$$

where one recognizes the ingoing and outgoing modes, propagating with the velocity $c=\eta^{-1}$, in agreement with the expression (3.38) obtained in the previous section, via the Schrödinger-like equation. 


\section{Numerical computation of the quasi-normal modes}

A very useful application of knowing the asymptotic solutions at infinity and near the horizon is the numerical computation of the quasi-normal modes (see e.g. the reviews [1 3]), as illustrated in Paper I for Schwarzschild black holes in General Relativity. In the context of modified gravity, quasi-normal modes have been computed explicitly for a few solutions, such as black holes in Einstein-Gauss-Bonnet [67 70] or dynamical Chern-Simons gravity [71.

Let us briefly explain the principle of the computation, based on [72], and apply it to the BCL solution. The asymptotic behaviour of the original metric variables $Y_{1}$ and $Y_{2}$, defined in (3.6), can be deduced from the asymptotic solutions to the diagonalised systems and the transition matrices. At spatial infinity, we have $Y=P \tilde{Y}$ where $P$ is given in (4.6), and the asymptotic behaviour (4.8) for $\tilde{Y}$ implies

$$
\begin{aligned}
& Y_{1}=\left(a_{+}^{\infty} r^{i \omega r_{\mathrm{m}}} e^{+i \omega r}-a_{-}^{\infty} r^{-i \omega r_{\mathrm{m}}} e^{-i \omega r}\right)(r+\mathcal{O}(1)), \\
& Y_{2}=\left(a_{+}^{\infty} r^{i \omega r_{\mathrm{m}}} e^{+i \omega r}+a_{-}^{\infty} r^{-i \omega r_{\mathrm{m}}} e^{-i \omega r}\right)(r+\mathcal{O}(1)),
\end{aligned}
$$

where, for later convenience, we have chosen the formulation in terms of $r$. A quasi-normal mode is characterized by purely outgoing boundary conditions at infinity, i.e.

$$
a_{-}^{\infty}=0 .
$$

At the horizon, the relation between the initial and final quantities is $Y=P_{(1)} P_{(2)} Y^{(2)}$, where $P_{(1)}$ and $P_{(2)}$ are defined in 4.15) and 4.17) respectively. The asymptotic solution 4.19) thus yields

$$
\begin{aligned}
& Y_{1}=\omega\left(a_{-}^{\text {hor }} \varepsilon^{-i \omega r_{0}}-a_{+}^{\text {hor }} \varepsilon^{+i \omega r_{0}}\right)(1+\mathcal{O}(\varepsilon)), \\
& Y_{2}=r_{0}\left(a_{-}^{\text {hor }} \varepsilon^{-i \omega r_{0}-1}+a_{+}^{\text {hor }} \varepsilon^{+i \omega r_{0}-1}\right)(1+\mathcal{O}(\varepsilon)) .
\end{aligned}
$$

For a quasi-normal mode, the boundary condition at the horizon must be purely ingoing, which requires

$$
a_{+}^{\text {hor }}=0 \text {. }
$$

Now we proceed as in Paper I to compute numerically the first quasi-normal modes of the axial perturbations about the BCL black hole. We first introduce an ansatz for $Y_{1}$ and $Y_{2}$, which satisfies the required boundary conditions,

$$
Y_{1}=e^{i \omega r} r^{1+i \omega r_{\mathrm{m}}}\left(\frac{r-r_{+}}{r}\right)^{-i \omega r_{0}} f_{1}(r), \quad Y_{2}=e^{i \omega r} r^{1+i \omega r_{\mathrm{m}}}\left(\frac{r-r_{+}}{r}\right)^{-1-i \omega r_{0}} f_{2}(r),
$$

where the functions $f_{1}$ and $f_{2}$ are supposed to be regular in the whole domain $\left[r_{+}, \infty\right.$ [ and bounded at spatial infinity and at the horizon. To implement these regularity conditions, we change the coordinate variable by setting

$$
u=\frac{2 r_{+}}{r}-1 \in[-1,+1],
$$

and decompose $f_{1}(u)$ and $f_{2}(u)$ onto the Chebyshev polynomials $T_{n}(u)$. We truncate the decomposition at a given order $N$, hence we have

$$
f_{1}(u)=\sum_{n=0}^{N} \alpha_{n} T_{n}(u), \quad f_{2}(u)=\sum_{n=0}^{N} \beta_{n} T_{n}(u),
$$


where $\alpha_{n}$ and $\beta_{n}$ are the complex coefficients to be determined by the resolution of the equations of motion. The next step consists in reformulating the differential system (3.7) as a system of linear algebraic equations of the form,

$$
M_{N}(\omega) V_{N}\left(\alpha_{n}, \beta_{n}\right)=0,
$$

where $M_{N}$ is a $2(N+1) \times 2(N+1)$ complex-valued matrix whose (finite) expansion in powers of $\omega$ read 13

$$
M_{N}(\omega)=M_{N[0]}+M_{N[1]} \omega+M_{N[2]} \omega^{2},
$$

while the $2(N+1)$-dimensional column vector $V_{N}\left(\alpha_{n}, \beta_{n}\right)$ contains the coefficients of the decompositions 4.30

$$
{ }^{T} V_{N}\left(\alpha_{n}, \beta_{n}\right) \equiv\left(\alpha_{0}, \cdots, \alpha_{N}, \beta_{0}, \cdots, \beta_{N}\right)
$$

Following [72], we transform the problem of solving the previous linear system in terms of a generalised eigenvalue problem which is formulated as follows,

$$
\tilde{M}_{N}(\omega) \tilde{V}_{N}\left(\alpha_{n}, \beta_{n}\right)=0,
$$

where the dimensions of the matrix $\tilde{M}_{N}$ and the vector $\tilde{V}_{N}$ have been doubled compared to the previous (untilded) ones according to

$$
\tilde{M}_{N}=\tilde{M}_{N[0]}+\tilde{M}_{N[1]} \omega \quad \text { and } \quad \tilde{M}_{N[0]}=\left(\begin{array}{cc}
M_{N[0]} & M_{N[1]} \\
0 & \mathbf{1}
\end{array}\right), \quad \tilde{M}_{N[1]}=\left(\begin{array}{cc}
0 & M_{N[2]} \\
-\mathbf{1} & 0
\end{array}\right) .
$$

At this stage, it is finally possible to compute the values of $\omega$ using Mathematica or Scipy. To proceed, we computed the modes for two different values of $N$ and kept the ones that agree up to a given precision, which allows us to get rid of the spurious solutions. The first quasi-normal modes have been represented in Fig. 2, We have also plotted, in Fig. 3, the "evolution" in the complex plane of the first three modes $(n=0,1,2)$ for $\ell=2,3$ when $\xi$ increases. One observes a decrease of both the real and (absolute value of the) imaginary parts of the complex frequencies as $\xi$ increases.

\section{Stealth Schwarzschild axial perturbations}

Let us now study the asymptotic behaviour of axial perturbations for the stealth Schwarzschild solution. As we saw in section IIIC, the dynamics of axial perturbations is now governed by the system (3.7),

$$
\frac{\mathrm{d} Y}{\mathrm{~d} r}=M Y, \quad M(r)=\left(\begin{array}{cc}
2 / r+i \omega \Psi & -i \omega^{2}+2 i \lambda \Phi / r^{2} \\
-i \Gamma & \Delta+i \omega \Psi
\end{array}\right)
$$

where the functions $\Psi, \Phi, \Gamma$ and $\Delta$ are given in (3.21). Let us also recall that the constant $\zeta(3.22)$ parametrizes the deviation to General Relativity which is recovered in the limit $\zeta \rightarrow 0$.

Following our remark, at the end of section IIIC, that the Schrödinger-like equation for axial modes is equivalent to a standard Regge-Wheeler equation, we now show that this property can be seen directly with the first order system, via appropriate rescalings of the time and radial variables.

${ }^{13}$ We use indices inside brackets to indicate the coefficients of the powers of $\omega$ (in contrast with the coefficients in the asymptotic expansions at spatial infinity or near the horizon). 


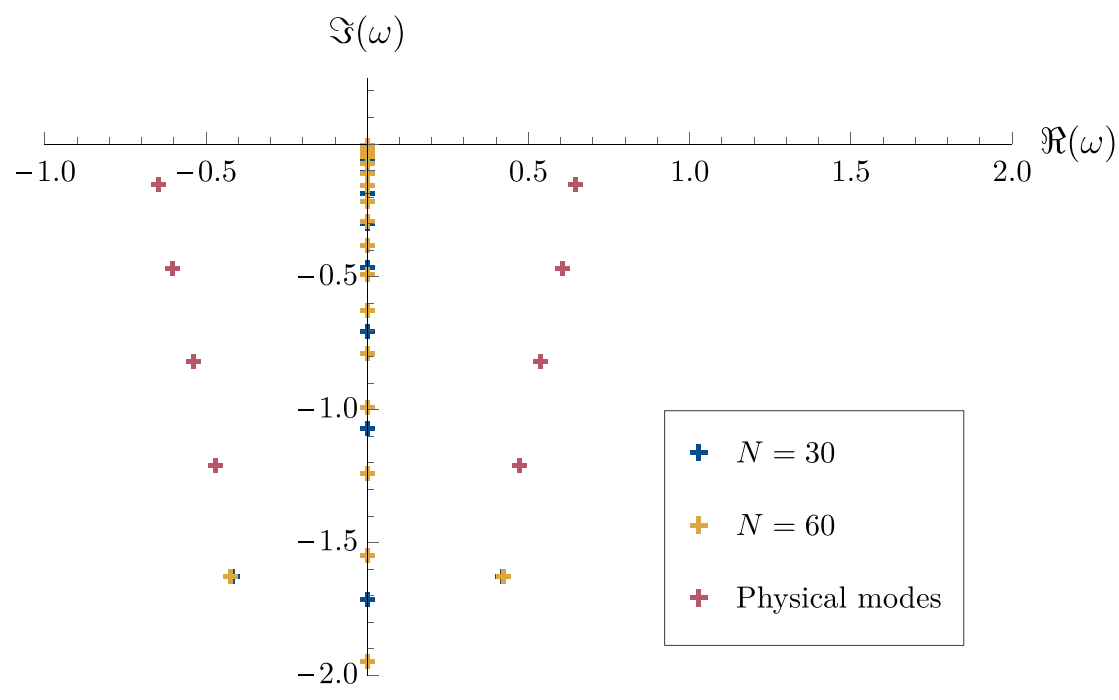

FIG. 2 Quasinormal modes numerically found for $\xi=0.5, r_{\mathrm{m}}=1$ and $\ell=2$. We take $N=30$, then $N=60$, and keep the values that agree up to $10^{-3}$. The eigenvalues shown in red correspond to the physical quasinormal modes, whereas the eigenvalues visible in blue or orange correspond either to spurious modes (on the imaginary axis) or to modes that have not yet converged. We can observe that there is a symmetry about the imaginary axis. The first three modes detected are $\omega_{0}= \pm 0.646-0.152 i, \omega_{1}= \pm 0.605-0.468 i$ and $\omega_{2}= \pm 0.534-0.819 i$.

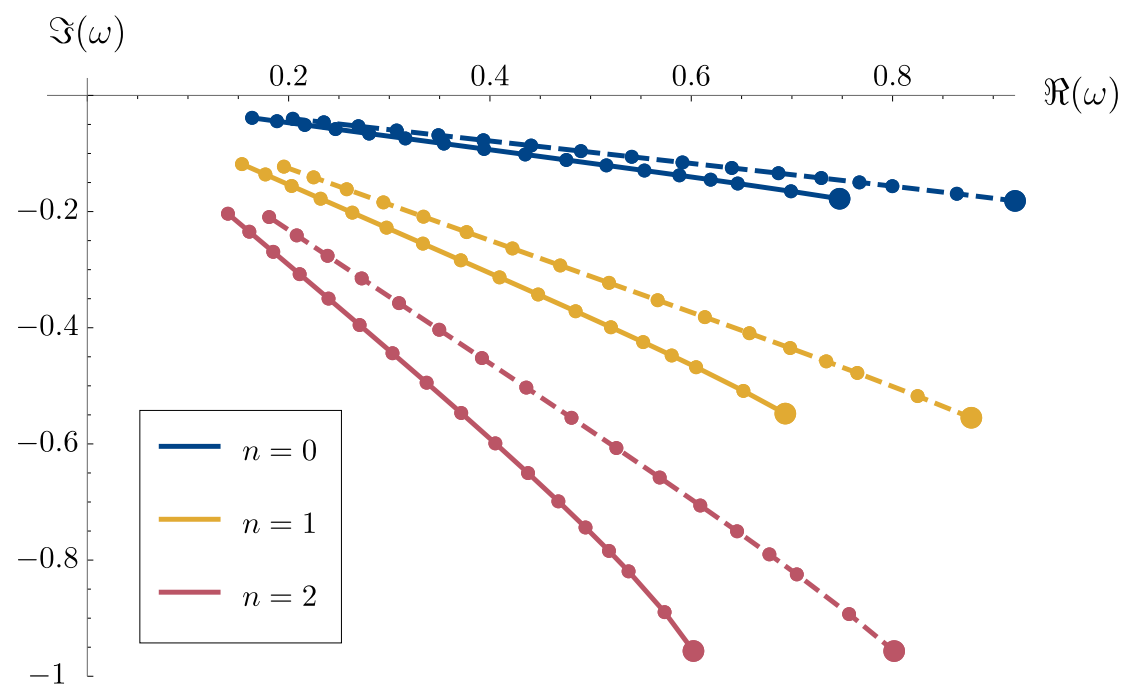

FIG. 3 The first three quasinormal modes $(n=0,1,2)$ for $\ell=2$ (continuous line) and $\ell=3$ (dashed line), with $r_{\mathrm{m}}=1$, when $\xi$ varies from 0 to 50 . On each "trajectory", the large dot denotes the GR mode $(\xi=0)$ and the next point corresponds to $\xi=0.2$, the subsequent values of $\xi$ increasing with a constant logarithmic increment until the final value $\xi=50$.

We first perform a time shift (3.17) with $\nu^{\prime}=-\Psi$ so that $\Psi$ disappears from the above matrix $M(r)$ in 4.36. Then, introducing the new variables

$$
\tilde{r} \equiv(1+\zeta) r, \quad \tilde{r}_{g} \equiv(1+\zeta) r_{g}, \quad \tilde{t} \equiv \sqrt{1+\zeta} t \Longrightarrow \tilde{\omega}=\omega / \sqrt{1+\zeta},
$$

one can see that the first order differential system takes exactly the same form as in GR, namely

$$
\frac{\mathrm{d} Y}{\mathrm{~d} \tilde{r}}=\tilde{M} Y, \quad \tilde{M}(\tilde{r})=\left(\begin{array}{cc}
2 / \tilde{r} & -i \tilde{\omega}^{2}+2 i \lambda \frac{\tilde{r}-\tilde{r}_{g}}{\tilde{r}^{3}} \\
-i \frac{\tilde{r}^{2}}{\left(\tilde{r}-\tilde{r}_{g}\right)^{2}} & -\frac{\tilde{r}_{g}}{\tilde{r}\left(\tilde{r}-\tilde{r}_{g}\right)}
\end{array}\right)
$$


with $\tilde{r}_{g}$ as Schwarzschild radius.

As a consequence, the asymptotic behaviour of $Y$ is immediately deduced from the GR results given in Paper I (section III.B). Both at infinity and near the horizon, the asymptotic behaviours of the two components of $Y$ are linear combinations (with coefficients that can depend on real powers of $r$ or $\varepsilon$ ) of the following outgoing and ingoing modes,

$$
e^{ \pm i \tilde{\omega} \tilde{r}_{*}}=e^{ \pm i \omega r_{*}}, \quad \tilde{r}_{*} \equiv \tilde{r}+\tilde{r}_{g} \ln \left(\tilde{r} / \tilde{r}_{g}-1\right)
$$

where $\tilde{r}_{*}$ corresponds to the standard tortoise coordinate in Schwarzschild (with radial coordinate $\tilde{r}$ and horizon $\tilde{r_{g}}$ ) and $r_{*}$ is the radial coordinate introduced in $(3.27)$ in order to get $c(r)=1$.

One can finally reintroduce the time dependence, taking into account the time shift $\nu$, to obtain the asymptotic limits. At spatial infinity, using $\nu(r)=-\int \Psi(r) d r \approx-2 \zeta \sqrt{r_{s} r}$, one finds

$$
e^{-i \omega(t+\nu)} e^{ \pm i \omega r_{*}} \approx e^{-i \omega(t+\nu)} e^{ \pm i \omega \sqrt{1+\zeta}\left(r+(1+\zeta) r_{s} \ln r\right)} \approx e^{-i \omega t} e^{2 i \omega \zeta \sqrt{r_{s} r}} e^{ \pm i \omega \sqrt{1+\zeta} r} r^{ \pm i \omega(1+\zeta)^{3 / 2} r_{s}}
$$

At the horizon $r=r_{g}$, using $\nu \approx-(1+\zeta)^{3 / 2} r_{\mathrm{s}} \ln \left(r / r_{g}-1\right) \approx-r_{*}$, one gets

$$
e^{-i \omega(t+\nu)} e^{ \pm i \omega r_{*}} \longrightarrow e^{-i \omega\left(t-2 r_{*}\right)} \text { and } e^{-i \omega t} .
$$

In the original coordinate system, only one mode seems to be propagating at the horizon. It is necessary to use a more appropriate time coordinate to identify one outgoing and one ingoing mode. The above expressions could also be obtained by applying the algorithm of Paper I to the original system.

\section{POLAR PERTURBATIONS}

We now turn to the study of polar, or even-parity, perturbations. We choose the same (Zerilli) gauge fixing as usually adopted in General Relativity (see e.g. Paper I for details), thus the metric perturbations are parametrised by four families of functions $H_{0}^{\ell m}, H_{1}^{\ell m}, H_{2}^{\ell m}$ and $K^{\ell m}$ ( $\ell$ and $m$ are integers with $\ell \geq 0$ and $-\ell \leq m \leq \ell$ ) such that the non-vanishing components of the metric are

$$
\begin{aligned}
& h_{t t}=A(r) \sum_{\ell, m} H_{0}^{\ell m}(t, r) Y_{\ell m}(\theta, \varphi), \quad h_{r r}=B(r)^{-1} \sum_{\ell, m} H_{2}^{\ell m}(t, r) Y_{\ell m}(\theta, \varphi), \\
& h_{t r}=\sum_{\ell, m} H_{1}^{\ell m}(t, r) Y_{\ell m}(\theta, \varphi), \quad h_{a b}=\sum_{\ell, m} K^{\ell m}(t, r) g_{a b} Y_{\ell m}(\theta, \varphi),
\end{aligned}
$$

where the indices $a, b$ belong to $\{\theta, \varphi\}$. The scalar field perturbation is parametrised by one family of functions according to

$$
\delta \phi=\sum_{\ell, m} \delta \phi^{\ell m}(t, r) Y_{\ell m}(\theta, \varphi)
$$

In the following we will consider only the modes $\ell \geq 2$ (the monopole $\ell=0$ and the dipole $\ell=1$ require different gauge fixing conditions).

We will study successively the BCL and stealth Schwarzschild solutions. Essentially, we proceed as in the previous section for axial perturbations. The main difference is that the first order system is now four-dimensional since it contains a scalar mode and a gravitational mode, which are coupled. By contrast with the axial case, we have not been able to reduce the system to a 2-dimensional Schrödinger-like equation, so the only option available to us in this case is the asymptotic analysis of the first-order system. We thus use the algorithm of Paper I to obtain the behaviour of the solutions of the system near the horizon and at spatial infinity. Since the calculations are more involved than in the axial case, we have summarised the steps of the procedure in the main text and confined the details to Appendix E. 


\section{A. BCL solution}

In the frequency domain, the linear equations of motion can be written as a four-dimensional first-order differential system (see Appendix E for details)

$$
\frac{\mathrm{d} Y}{\mathrm{~d} r}=M Y
$$

with the column vector

$$
Y={ }^{T}\left(K \chi H_{1} H_{0}\right)
$$

where $\chi$ corresponds to a renormalised scalar field perturbation, namely

$$
\chi(r) \equiv \frac{f_{1}}{f_{0} \sqrt{A(r)}} \delta \phi(r) .
$$

The explicit form of the square matrix $M$ can be read off from the equations of motion (see discussion in Appendix C 2 )

$$
M=\left(\begin{array}{cccc}
-\frac{1}{r}+\frac{\mathcal{U}}{2 r^{3} A} & \frac{\mathcal{U}}{r^{4}} & \frac{i(1+\lambda)}{\omega r^{2}} & \frac{\mathcal{V}}{r^{3}} \\
\frac{\omega^{2} r^{2}}{A^{2}}-\frac{\lambda}{A}-\frac{r_{m}}{2 r A}+\frac{r_{\mathrm{m}}^{2} \mathcal{S}}{4 r^{4} A^{2}} & -\frac{2}{r}-\frac{\mathcal{U} \mathcal{V}}{2 r^{5} A} & -\frac{i \omega r}{A}+\frac{i(1+\lambda) \mathcal{U}}{2 r^{3} \omega A} & -\frac{\lambda}{A}-\frac{3 \mathcal{U}}{2 r^{3} A}-\frac{\xi^{2} r_{\mathrm{m}}^{4}}{2 r^{4} A} \\
-\frac{i \omega \mathcal{V}}{r^{2} A} & \frac{2 i \omega}{r}-\frac{i \omega \mathcal{U}}{r^{3} A} & -\frac{\mathcal{U}^{3} A}{r^{3} A} & -\frac{i \omega \mathcal{V}}{r^{2} A} \\
-\frac{1}{r}+\frac{\mathcal{U}}{2 r^{3} A} & \frac{2}{r^{2}}-\frac{\mathcal{U}^{2}}{2 r^{6} A} & -\frac{i \omega}{A}+\frac{i(1+\lambda)}{\omega r^{2}} & \frac{1}{r}-\frac{\mathcal{U}}{2 r^{3} A}-\frac{\mathcal{U} \mathcal{V}}{2 r^{5} A}
\end{array}\right)
$$

where we have introduced the functions

$$
\mathcal{U}(r) \equiv r_{\mathrm{m}}\left(r+\xi r_{\mathrm{m}}\right), \quad \mathcal{V}(r) \equiv r^{2}+\xi r_{\mathrm{m}}^{2}, \quad \mathcal{S}(r) \equiv r^{2}+2 \xi r\left(2 r_{\mathrm{m}}-r\right)+2 \xi^{2} r_{\mathrm{m}}^{2} .
$$

We analyse below the asymptotic behaviours of the above system, first at spatial infinity and then near the horizon.

\section{At spatial infinity}

The expansion of the matrix $M$ in $(5.6)$ at spatial infinity is of the form

$$
M(r)=r^{2} M_{2}+r M_{1}+M_{0}+\frac{1}{r} M_{-1}+\mathcal{O}\left(\frac{1}{r^{2}}\right),
$$

where the matrices $M_{i}$ can easily be inferred from (5.6).

The leading matrix $M_{2}$ contains a single non-zero entry, $\left(M_{2}\right)_{21}=\omega^{2}$, and is thus nilpotent. To diagonalise the system, one can follow step by step the algorithm presented in Paper I. Here, however, in order to shorten the procedure, we first adopt a "customised" strategy by considering a transformation of the form

$$
Y=P_{(1)} Y^{(1)}, \quad P_{(1)}=\operatorname{Diag}\left(r^{p_{1}}, r^{p_{2}}, r^{p_{3}}, r^{p_{4}}\right)
$$

and choosing the powers $p_{i}$ that simplify the system the most. With the choice

$$
p_{1}=0, \quad p_{2}=2, \quad p_{3}=p_{4}=1,
$$

one finds that the system becomes

$$
\frac{\mathrm{d} Y^{(1)}}{\mathrm{d} r}=M^{(1)} Y^{(1)}, \quad M^{(1)}=M_{0}^{(1)}+\frac{1}{r} M_{-1}^{(1)}+\mathcal{O}\left(\frac{1}{r^{2}}\right),
$$


where the two matrices $M_{0}^{(1)}$ and $M_{-1}^{(1)}$ have the simple expressions

$$
M_{0}^{(1)}=\left(\begin{array}{cccc}
0 & 0 & 0 & 1 \\
-\omega^{2} & 0 & i \omega & 0 \\
0 & -2 i \omega & 0 & -i \omega \\
0 & 0 & -i \omega & 0
\end{array}\right), \quad M_{-1}^{(1)}=\left(\begin{array}{cccc}
-1 & -r_{\mathrm{m}} & i(1+\lambda) / \omega & 0 \\
-2 \omega^{2} r_{\mathrm{m}} & -4 & 0 & -\lambda \\
-i \omega & i \omega r_{\mathrm{m}} & -1 & -i \omega r_{\mathrm{m}} \\
0 & -2 & -i \omega r_{\mathrm{m}} & 0
\end{array}\right)
$$

Following now the algorithm of Paper I, two additional steps are needed to obtain a fully diagonalised system (up to order $r^{0}$ ), given by

$$
\frac{\mathrm{d} \tilde{Y}}{\mathrm{~d} r}=\tilde{M} \tilde{Y}, \quad(Y=\tilde{P} \tilde{Y})
$$

where the (combined) transition matrix $\tilde{P}$ and the expansion of $\tilde{M}$ are given explicitly in Appendix E. Integrating this asymptotic system yields

$$
\tilde{Y}(r)=\left(\begin{array}{c}
c_{-} r^{-i \omega r_{\mathrm{m}}} e^{-i \omega r} \\
c_{+} r^{+i \omega r_{\mathrm{m}}} e^{+i \omega r} \\
\frac{d_{-}}{r^{3}} r^{-\frac{\omega r_{\mathrm{m}}}{\sqrt{2}}} e^{-\sqrt{2} \omega r} \\
\frac{d_{+}}{r^{3}} r^{+\frac{\omega r_{\mathrm{m}}}{\sqrt{2}}} e^{+\sqrt{2} \omega r}
\end{array}\right)(1+\mathcal{O}(1 / r))
$$

where $c_{ \pm}$and $d_{ \pm}$are constants.

The first two components are very similar to the components of the asymptotic solution obtained in the axial sector (see (4.8) ) and it is therefore natural to identify these modes with the usual outgoing and ingoing gravitational modes. By contrast, the last two components have an unusual form. If we return to the original variables, via the transformation (E1), we find that the asymptotic behavior of the (renormalized) scalar perturbation $\chi(5.5)$ reads

$$
\chi(r)=\frac{3}{2 r}\left[d_{-} r^{-\frac{\omega r_{m}}{\sqrt{2}}} e^{-\sqrt{2} \omega r}-d_{+} r^{\frac{\omega r_{m}}{\sqrt{2}}} e^{\sqrt{2} \omega r}\right](1+\mathcal{O}(1 / r)) .
$$

The behaviour exhibited by this perturbation appears problematic, as it is associated with an effective metric which does not possess the appropriate causal structure. Indeed, the asymptotic solution 5.15 can be related to an equation of motion for $\tilde{\chi} \equiv r \chi$ of the form

$$
\frac{\partial^{2} \tilde{\chi}}{\partial t^{2}}+\frac{\partial^{2} \tilde{\chi}}{\partial \tilde{r}^{2}} \approx 0, \quad \text { with } \quad \tilde{r}=\sqrt{2}\left(r+\frac{r_{\mathrm{m}}}{2} \ln r\right)
$$

which does not correspond to a wave equation. This non-hyperbolicity is usually associated with a ghost or gradient instability.

For a more direct - although less rigorous - approach to this problem, it is instructive to examine the perturbations of the scalar field on the fixed background geometry, in other words to ignore the backreaction of the scalar field perturbations on the metric. In this case, the equation of motion for the scalar field perturbation $\chi$ is of the form

$$
\frac{\partial^{2} \chi}{\partial t^{2}}+\frac{1}{2} A(r) \frac{\partial^{2} \chi}{\partial r^{2}}+\frac{1}{r}\left(1+\frac{\xi r_{\mathrm{m}}^{2}}{2 r^{2}}\right) \frac{\partial \chi}{\partial r}-W(r) \chi=0
$$

where $W(r)$ is some potential, given explicitly in Appendix G. Since $A>0$, this equation has the structure of an elliptic equation, similar to (5.16). In fact, it is even possible to show that the asymptotic behaviour (5.15) can be directly recovered from (G2), as shown in Appendix G. 


\section{Near the horizon}

To obtain the asymptotic behaviour near the horizon, we define, as usual, the small parameter $\varepsilon \equiv r-r_{+}$. It is then convenient to make the following initial change of vector to simplify the analysis:

$$
Y=P_{(1)} Y^{(1)}, \quad P_{(1)}=\left(\begin{array}{cccc}
1 & 0 & 0 & 0 \\
0 & 1 / \varepsilon & 0 & 0 \\
0 & 0 & 1 / \varepsilon & 0 \\
0 & 0 & 0 & 1 / \varepsilon
\end{array}\right)
$$

The matrix $M^{(1)}$ associated to the system for $Y^{(1)}$ admits a very simple asymptotic expansion, of the form

$$
M^{(1)}=\frac{1}{\varepsilon} M_{0}^{(1)}+\mathcal{O}(1)
$$

where the matrix $M_{0}^{(1)}$ is given in $\mathrm{E} 6$ in the Appendix $\mathrm{E}$.

After transforming this matrix into a Jordan block form as shown in Appendix E, one finds that the asymptotic expansion of the modes reads

$$
Y^{(2)}(r)=\left(\begin{array}{c}
c_{-} \varepsilon^{-i \omega r_{0}} \\
c_{+} \varepsilon^{+i \omega r_{0}} \\
\left(a_{1} \ln \varepsilon+a_{2}\right) \sqrt{\varepsilon} \\
a_{1} \sqrt{\varepsilon}
\end{array}\right)(1+\mathcal{O}(\varepsilon))
$$

where again $c_{ \pm}, a_{1}$ and $a_{2}$ are constant. The correspondence between the original vector $Y$ and $Y^{(2)}$ and the expression of the matrix $P=P_{(1)} P_{(2)}$ are described in the Appendix $\mathrm{E}$.

The behaviour of the first two components in $(5.20)$ is the same as in the axial case, and one can thus identify them with the ingoing and outgoing gravitational modes. By contrast, the behaviour of the last two components is very peculiar and is related to the presence of the scalar field degree of freedom. As in the spatial infinity limit, these modes do not seem to correspond to a second-order equation respecting the usual four-dimensional causal structure, which indicates that the effective metric near the horizon, in which the perturbations propagate, is pathological.

\section{Computation of the quasinormal modes}

In the following, we restrict ourselves to the "gravitational" modes, which behave asymptotically like the axial modes. We do not consider the "scalar" modes, whose pathological behaviour probably indicates the presence of an instability, as mentioned earlier. To compute numerically the quasi-normal modes, we extend the method of section IVB 3 to a 4-dimensional system. At spatial infinity, we require the modes to be purely outgoing, while they must be purely ingoing near the horizon. This implies the restrictions

$$
c_{-}=d_{+}=d_{-}=0, \quad c_{+}=a_{1}=a_{2}=0,
$$


in (5.14) and (5.20), respectively. Taking into account these requirements, we consider the following ansätze for the four perturbations:

$$
\begin{aligned}
H_{0}(r) & =e^{i \omega r} r^{1+i \omega r_{\mathrm{m}}}\left(\frac{r-r_{+}}{r}\right)^{-1+i \omega r_{0}} f_{0}(r), \\
H_{1}(r) & =\omega e^{i \omega r} r^{1+i \omega r_{\mathrm{m}}}\left(\frac{r-r_{+}}{r}\right)^{-1+i \omega r_{0}} f_{1}(r), \\
K(r) & =e^{i \omega r} r^{i \omega r_{\mathrm{m}}}\left(\frac{r-r_{+}}{r}\right)^{+i \omega r_{0}} f_{K}(r), \\
\chi(r) & =e^{i \omega r} r^{-1+i \omega r_{\mathrm{m}}}\left(\frac{r-r_{+}}{r}\right)^{-1+i \omega r_{0}} f_{\chi}(r),
\end{aligned}
$$

where the functions $f_{0}, f_{1}, f_{K}$ and $f_{\chi}$ are supposed to be bounded.

Decomposing these functions onto Chebyshev polynomials, up to some order $N$, the differential system with (5.6) is transformed into the matricial equation

$$
M_{N}(\omega) V_{N}=0, \quad \text { with } \quad M_{N}(\omega)=M_{N[0]}+M_{N[1]} \omega+M_{N[2]} \omega^{2},
$$

where the components of the $4(N+1)$-dimensional column vector $V_{N}$ are the components of the functions $f_{0}, f_{1}, f_{K}$ and $f_{\chi}$ on the Chebyshev basis. Once again, this linear system corresponds to a generalised eigenvalue problem and the values of $\omega$ can be determined numerically. Changing the truncation order $N$ then enables us to identify the quasi-normal modes of the full system.

The first modes are represented in Fig. 4. Even though the numerical analysis could be further refined 14 , we can already make interesting observations. First, when the parameter $\xi$ vanishes, all the modes found agree with the ones of Schwarzschild in General Relativity as expected. When $\xi$ is not vanishing and increases, the real and imaginary parts of the modes decrease compared to those of GR. It is interesting to note that we have obtained a continuous deformation of the classical branch of the polar modes in GR and no other modes are detected. In other words, there is a one-to-one correspondance between the metric polar modes of the BCL black hole and the modes of the Schwarzschild black hole in GR. Hence, it seems that imposing the metric boundary conditions, recalled above, on the equations of perturbations is sufficient to ensure only the metric modes are computed.

${ }^{14}$ We can see that the results are plagued with a lot of spurious eigenvalues caused by numerical errors, which prevents us from probing higher values of $\xi$, or higher-overtone modes. This problem comes from the higher order of the coupled system: it is made of four first-order equations, while the system for axial modes involves only two equations. In order to get accurate estimates of the frequencies, we need to increase the precision of the computations, and this is extremely time-consuming. This is the reason why we do not probe higher-overtone modes here. 


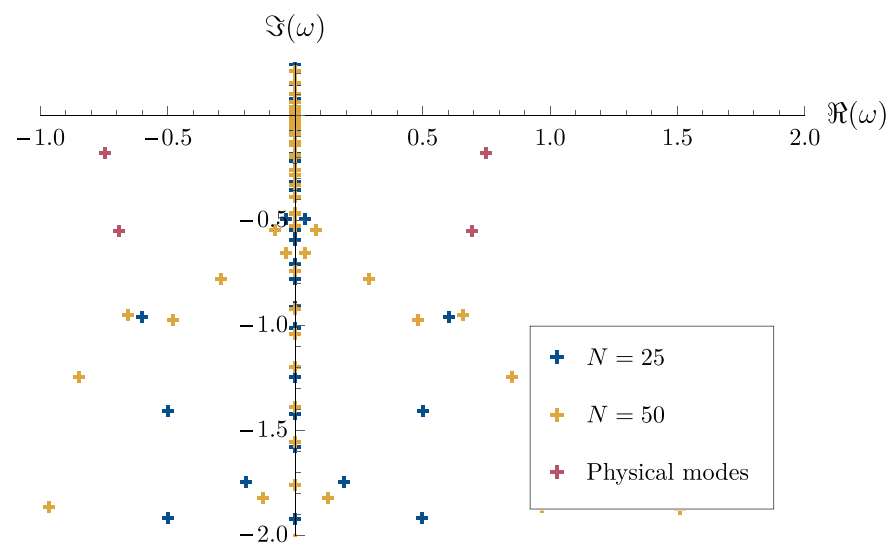

FIG. 4 Quasinormal modes found for $\xi=10^{-4}$ and $r_{\mathrm{m}}=1$. We take $N=25$, then $N=50$, and keep the values that agree up to $10^{-3}$. We can observe that there is a symmetry about the imaginary axis. Only the first two modes are detected, and they match with the Schwarzschild frequencies up to $10^{-3}$.

As a final but interesting remark, we underline that the well-known degeneracy between axial and polar modes (the so-called iso-spectrality property) in GR is lifted when one considers the BCL solution. Indeed, the polar and axial modes associated to the same overtone are different as soon as $\xi \neq 0$. This is illustrated in Fig. 5. Such a feature could be used to discriminate between a GR black hole and a modified gravity black hole in the ringdown phase of a black hole merger.

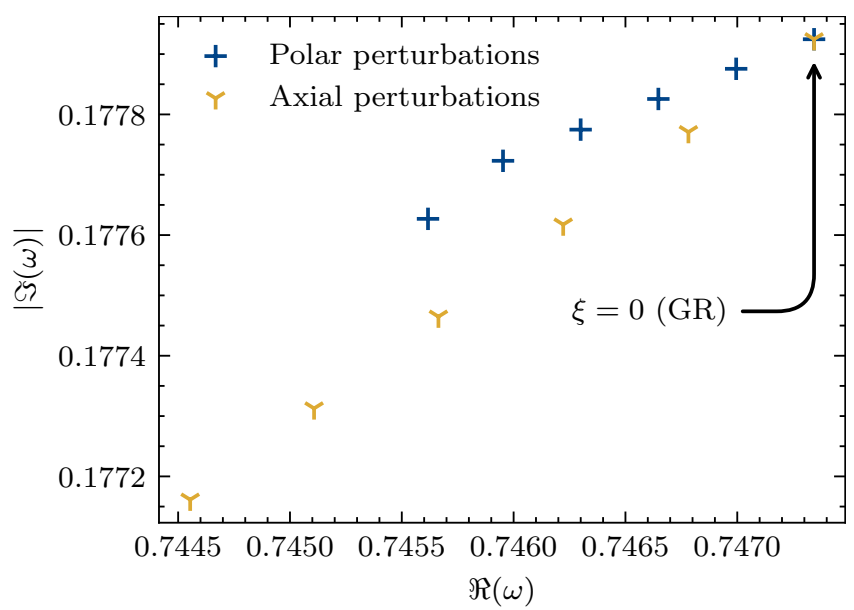

FIG. 5 Tracking of the fundamental metric mode of BCL for $0 \leq \xi \leq 0.01$. The parameter $\xi$ is increased by 0.002 between each point. We observe that the polar and axials modes are identical in the GR limit (as expected), but become different as soon as $\xi \neq 0$.

\section{B. Stealth Schwarzschild solution}

The asymptotic behaviour of polar perturbations for stealth Schwarzschild can be computed with the same procedure as in the BCL case, even if it turns out to be technically more involved, with rather tedious calculations. Since the details are not very illuminating, we simply give the final results in this section. Furthermore, to simplify the analysis, we will consider theories where only one of the parameters $\alpha, \beta$ or $\gamma$ defined in 2.9 is non zero.

In each case, we find that the asymptotic expansion of the four-dimensional column vector $Y(r)$ can be written as a linear combination of four modes, which we will denote $\mathfrak{g}_{ \pm}(r)$ for the modes 
analogous to the axial gravitational modes and $\mathfrak{s}_{ \pm}(r)$ for the additional modes. There will be two families of such modes, one at spatial infinity and the other one near the horizon, which will be distinguished by the subscript $\infty$ or h, respectively. We give below the leading order behaviour of the modes, ignoring possible multiplicative factors that are powers of $r$ or of $\varepsilon \equiv r-r_{\mathrm{s}}$ with a real exponent.

For the theories with $\beta \neq 0$ or $\gamma \neq 0$, we find the following common behaviours:

- at spatial infinity:

$$
\mathfrak{g}_{ \pm}^{\infty}(r) \approx r^{ \pm i \omega r_{\mathrm{s}}} e^{ \pm i \omega r}
$$

- near the horizon:

$$
\mathfrak{g}_{ \pm}^{\mathrm{h}}(\varepsilon) \approx \varepsilon^{ \pm i \omega r_{\mathrm{s}}}, \quad \mathfrak{s}_{ \pm}^{\mathrm{h}}(\varepsilon) \approx \varepsilon^{-i \omega r_{\mathrm{s}}}
$$

By contrast, the behaviours of the "scalar" modes at spatial infinity are different in the two cases:

$$
\beta \neq 0: \quad \mathfrak{s}_{ \pm}^{\infty}(r) \approx e^{-2 i \omega r_{\mathrm{s}} z} z^{ \pm 2 i \sqrt{\lambda}}, \quad \gamma \neq 0: \quad \mathfrak{s}_{ \pm}^{\infty}(r) \approx e^{-2 i \omega r_{\mathrm{s}} z\left(z^{2} / 3+1\right)},
$$

where we recall that $z \equiv \sqrt{r / r_{\mathrm{s}}}$.

One observes that, in some cases, the + and - modes share exactly the same leading behaviour at spatial infinity or near the horizon. As a consequence, the usual distinction between ingoing and outgoing modes becomes difficult, at least at leading order, and might require to consider the next orders in the asymptotic expansion. It is also worth noting that, in the cases $\gamma \neq 0$ and $\beta \neq 0$, the equations for the perturbations drastically simplify, as shown in Appendix $\mathrm{F}$ for $\gamma \neq 0$, and the asymptotic behaviour of the scalar field can be obtained from the perturbed conservation equation

$$
\nabla_{\mu}\left(\delta X \phi^{\mu}\right)=\frac{1}{\sqrt{-g}} \partial_{\mu}\left(\sqrt{-g} \delta X \phi^{\mu}\right)=0,
$$

where $g_{\mu \nu}$ is the Schwarzschild metric and $\delta X$ is the perturbation of $X=\phi_{\mu} \phi^{\mu}$. Remarkably this equation can be solved explicitly (at least in the case $\gamma \neq 0$ ) and its solution reproduces exactly the asymptotic behaviour of the scalar field derived from the analysis of the first order system.

Finally, in the case $\alpha \neq 0$, we find the following asymptotic behaviours at spatial infinity:

$$
\mathfrak{g}_{ \pm}^{\infty}(r) \approx e^{ \pm i \omega r_{*}+2 i \omega \zeta \sqrt{r_{\mathrm{s}} r}}, \quad \mathfrak{s}_{ \pm}^{\infty}(r) \approx e^{-2 i \omega r_{\mathrm{s}} z},
$$

where $r_{*}$ is the coordinate introduced in (3.27). For the "gravitational" modes, one can clearly identify the ingoing and outgoing modes, and the term proportional to $\sqrt{r_{\mathrm{s}} r}$ in the exponential of $\mathfrak{g}_{ \pm}^{\mathrm{h}}(r)$ could be absorbed by a time redefinition of the form (3.17). At the horizon, the study of the asymptotic behaviour is more subtle because in that case the "scalar" modes and the "gravitational" modes might "see" different horizons.

We will restrict our discussion here to the horizon $r_{g}$, where the axial modes behave as in GR as we have seen. Near $r=r_{g}$, we find

$$
\mathfrak{g}_{+}^{\mathrm{h}}(\varepsilon) \approx \varepsilon^{2 i \omega(1+\zeta)^{3 / 2} r_{\mathrm{s}}} \approx e^{2 i \omega r_{*}} \quad \text { and } \mathfrak{g}_{-}^{\mathrm{h}}(\varepsilon) \approx 1, \text { where } \varepsilon \equiv r-r_{g} .
$$

We thus recover exactly the same behaviour as for the axial modes obtained in (4.41). Performing the same time shift detailed in (3.19), the above modes in (5.29) would become

$$
\mathfrak{g}_{ \pm}^{\mathrm{h}}(\varepsilon) \approx e^{ \pm i \omega r_{*}}
$$


which can be interpreted as ingoing and outgoing modes. In summary, the polar and axial "gravitational" modes have similar asymptotic properties, which are more easily interpreted in the effective metric with horizon at $r=r_{g}$. We leave a detailed study of the behaviour of the "scalar" modes for a future work.

As mentioned in the introduction, a detuning of the degeneracy conditions, called "scordatura", was proposed in [30] as a solution to the strong coupling problem of the stealth solutions. In order to include this type of model, the method developed here would need to be extended. Indeed, if the degeneracy conditions are not satisfied, the perturbation system contains higher order equations. They can nevertheless be recast into a higher-dimensional first-order system, to which we can apply our method. We leave the study of these models for future work.

\section{CONCLUSION}

In this article, we have applied the novel approach introduced in Paper I to study linear black hole perturbations in the context of DHOST theories. The method is very generic and enables one to obtain the asymptotic behaviours of the perturbations at spatial infinity and near the black hole horizon without reformulating their dynamics in terms of a Schrödinger-like equation. The knowledge of these asymptotic behaviours is essential to define and compute the quasi-normal modes, characterised by outgoing conditions at spatial infinity and ingoing conditions at the horizon.

We have considered here two examples of nonrotating black hole solutions within DHOST theories. The first one is the Schwarzschild stealth solution whose geometry is described by the usual Schwarzschild metric while the second one is the non-stealth BCL solution whose metric is analogous to that of Reissner-Nordstrom black hole with the square of the electric charge effectively negative. In both cases, the scalar field has a non-trivial profile (but $X=\phi_{\mu} \phi^{\mu}$ is constant in the former case whereas it depends on the radial coordinate $r$ in the latter case).

We have treated separately axial and polar perturbations. Since the scalar field perturbation is polar, axial perturbations are described by a single (gravitational) degree of freedom and are thus easier to study. In particular, their equations of motion can be reformulated as a Schrödinger-like equation and we have found a simple method to compute explicitly the corresponding effective potential (which depends on the choice of the radial coordinate, as the propagation speed does). For some stealth solutions, one obtains the very peculiar property that the axial modes "see" a Schwarzschild metric with a displaced horizon, corresponding to the disformal transformation of the original metric into the "frame" where the propagation speed is unity.

For axial perturbations of the BCL solution, instead of computing the quasi-normal modes in the traditional way by numerically solving the Schrödinger-like equation, we have used the novel method of Paper I. We have thus first computed the asymptotic behaviours of the perturbations, at infinity and near the horizon, from the original differential system. We have then computed, using a spectral method, the first quasi-normal modes for BCL, finding a deviation from general relativity.

The study of polar perturbations and the computation of the associated quasi-normal modes is more challenging because the scalar field and metric perturbations are now coupled and we have not found a generalised Schrödinger-like reformulation of the system. The only option left was thus to apply the method of Paper I, providing the asymptotic behaviours of the solutions at spatial infinity and near the horizon for both types of black holes. For the BCL solution, we have identified two pairs of modes at the boundaries. One pair consists of an ingoing mode and an outgoing mode, which look similar to the usual gravitational modes. By contrast, the other two modes, corresponding to "scalar" modes, possess an asymptotic behaviour that appears pathological. Restricting ourselves to the "gravitational modes", we have computed numerically the 
first quasi-normal modes for the BCL solution, both for axial and polar modes. They are distinct from the GR Schwarzschild quasi-normal modes and, as expected, the isospectrality property breaks down as the polar and the axial quasi-normal modes are now different.

For the stealth black hole solution, we have found that the "gravitational" polar modes behave asymptotically as their axial counterparts. In the stealth models with $\alpha \neq 0$, their behaviour is similar to the standard GR behaviour but in a disformed Schwarzschild metric, with a different horizon and characterised by a radially-dependent time shift. The polar modes also contain two additional modes, due to the presence of the scalar field, for which we have computed some asymptotic limits. We leave for a future work a detailed analysis of these "scalar" modes.

This work opens a new window for the investigation of black hole perturbations in modified gravity. The potential of the new method presented in Paper I has been illustrated here with just a couple of examples and a rudimentary numerical treatment. We plan to develop it further in the future, especially the numerical approach in order to reach a precision that would be useful for observational constraints. We would also like to extend our investigation to other background solutions. Note that it would be interesting to explore the use of the asymptotic limits as a first diagnostic tool for potential pathologies of black hole solutions with scalar hair.

\section{ACKNOWLEDGMENTS}

We would like to thank Eugeny Babichev, Christos Charmousis and Gilles Esposito-Farèse for interesting discussions, as well as Emanuele Berti and Vitor Cardoso for instructive correspondence.

\section{Appendix A: Stealth solutions in DHOST theories}

In this Appendix, we recall and discuss the conditions for a DHOST theory to admit stealth solutions, i.e. solutions of modified gravity whose metric coincides with a vacuum solution of General Relativity plus a cosmological constant.

The main stealth solutions in shift-symmetric DHOST theories are described by the Schwarzschild metric and a scalar field of the form

$$
\phi(t, r)=q t+\psi(r)
$$

where $q$ is constant. We also assume a constant value for $X \equiv \phi_{\mu} \phi^{\mu}$, which we denote $X_{0}$.

Stealth Schwarzschild solutions can be found in DHOST theories, with either $X_{0}=-q^{2}$ or $X_{0} \neq-q^{2}$, provided that the functions appearing in the action (2.1) satisfy the conditions (see Eq. (22) of [15])

$$
\begin{aligned}
& P=P_{X}=Q_{X}=A_{1}+A_{2}=A_{1 X}+A_{2 X}=0, \\
& \left(X_{0}+q^{2}\right) A_{1}=\left(X_{0}+q^{2}\right)\left(2 A_{1 X}+A_{3}\right)=0 \quad\left(\text { at } X=X_{0}\right),
\end{aligned}
$$

where all functions are evaluated at $X=X_{0}$. These conditions were shown to be necessary and sufficient for the equations of motion of the metric to reduce to those of General Relativity for static and spherical symmetric metric [15]. Type Ia DHOST theories verify $A_{2}(X)=-A_{1}(X)$, which implies that the last two conditions in $(\mathrm{A} 2$ are automatically satisfied. By contrast, the conditions $\mathrm{A} 3$ are more restrictive if $X_{0}+q^{2} \neq 0$. These two cases were discussed in detail in [15.

One can also look for DHOST theories such that any solution of General Relativity (with a cosmological constant $\Lambda$ ), not only thee static spherically symmetric metric solutions, is also 
solution of the DHOST theory, which imposes much more stringent conditions [22]:

$$
P+2 \Lambda F=0, \quad P_{X}+\Lambda\left(4 F_{X}-X_{0} A_{1 X}\right)=0, \quad Q_{X}=0, \quad A_{1}=0 \quad A_{3}+2 A_{1 X}=0,
$$

where all these expressions are evaluated at $X=X_{0}$. These conditions have been recently generalised to non-shift symmetric theories and to the case where matter is coupled to gravity minimally [22].

\section{Appendix B: Background equations of motion}

The variation of the shift-symmetric Horndeski action (2.4) yields the equations of motion

$$
\mathcal{B}_{\mu \nu} \equiv \frac{\delta S}{\delta g_{\mu \nu}}=0, \quad \mathcal{B}_{\phi} \equiv \frac{\delta S}{\delta \phi}=0
$$

Due to Bianchi identities, the equation for the scalar field is not independent from the metric equations and therefore can be ignored.

For a metric of the form (2.6) and a scalar field profile (2.7), one finds that the only non-trivial equations are given, up to a global irrelevant factor, by

$$
\begin{aligned}
\mathcal{B}_{t t} \propto & \frac{1}{2} A P+q^{2} P_{X}-\frac{A}{r^{2}}\left(-1+B+r B^{\prime}\right) F+\frac{2 q^{2}}{r^{2}}\left(1-r B \frac{A^{\prime}}{A}\right) F_{X} \\
& +\frac{2 A}{r^{2}} \frac{\mathrm{d}(r X B)}{\mathrm{d} r} F_{X}-\frac{4 B}{r^{2} A}\left(q^{4}+q^{2} X\left(A+r A^{\prime}\right)-r A^{2} X X^{\prime}\right) F_{X X} \\
& +\frac{1}{2}\left[q^{2} B^{\prime} \psi^{\prime}+B \psi^{\prime}\left(\frac{4 q^{2}}{r}+\frac{q^{2} A^{\prime}}{A}-A X^{\prime}\right)+2 B q^{2} \psi^{\prime \prime}\right], \\
\mathcal{B}_{t r} \propto & q \psi^{\prime} P_{X}+\frac{q}{2 r}\left(\frac{4 q^{2}}{A}+4 X+r X \frac{A^{\prime}}{A}\right) Q_{X} \\
& -\frac{2 q \psi^{\prime}}{r^{2}}\left(B-1+r B \frac{A^{\prime}}{A}\right) F_{X}-\frac{4 q \psi^{\prime} B}{r^{2}}\left(\frac{q^{2}}{A}+X+r X \frac{A^{\prime}}{A}\right) F_{X X}, \\
\mathcal{B}_{r r} \propto & -\frac{1}{2 B} P+\frac{1}{r^{2}}\left(1-\frac{1}{B}+\frac{r A^{\prime}}{A}\right) F-\frac{2 q^{2}}{r^{2} A}\left(2-\frac{1}{B}+\frac{r A^{\prime}}{A}\right) F_{X} \\
& -\frac{4 X}{r^{2}}\left(1-\frac{2}{B}+\frac{r A^{\prime}}{A}\right) F_{X}+\left(\psi^{\prime}\right)^{2} P_{X}+\frac{\psi^{\prime}}{2 r}\left(\frac{4 q^{2}}{A}+4 X+r X \frac{A^{\prime}}{A}\right) Q_{X} \\
& -\frac{4}{r^{2}} B\left(\psi^{\prime}\right)^{2}\left(\frac{q^{2}}{A}+X+r X^{\prime}\right) F_{X X}, \\
\mathcal{B}_{\theta \theta} \propto & \mathcal{B}_{\varphi \varphi} \propto-\frac{1}{2} r^{2} P+\frac{1}{2} r^{2} B \psi^{\prime} X^{\prime} Q_{X}-\frac{B^{\prime} r}{2}\left(\frac{2 q^{2}}{A}+2 X+r X \frac{A^{\prime}}{A}\right) F_{X} \\
& +\frac{r}{4}\left[2 B^{\prime}-r B\left(\frac{A^{\prime}}{A}\right)^{2}+\frac{B}{A}\left(r \frac{A^{\prime}}{A} \frac{B^{\prime}}{B}+2 \frac{A^{\prime}}{A}+2 r \frac{A^{\prime \prime}}{A}\right)\right] F \\
& -\frac{r B A^{\prime}}{2 A}\left(-\frac{2 q^{2}}{A}+2 X+r X^{\prime}\right) F_{X}-r X^{\prime} B\left(\frac{2 q^{2}}{A}+2 X+r X \frac{A^{\prime}}{A}\right) F_{X X} \\
& \left.r B\left(\frac{A^{\prime}}{A}\right)^{2}+2\left(X^{\prime}+r X \frac{A^{\prime \prime}}{A}\right)\right],
\end{aligned}
$$

where a prime denotes a derivative with respect to $r$. $X$ is related to $A, B$ and $\psi^{\prime}$ by the equation

$$
X=g^{\mu \nu} \partial_{\mu} \phi \partial_{\nu} \phi=-\frac{q^{2}}{A}+B\left(\psi^{\prime}\right)^{2} .
$$


Assuming $X$ to be constant drastically simplifies the above metric equations. For a Schwarzschild metric, the equations admit a solution only if the stealth conditions A2) and (A3) (restricted to Horndeski theories) are fulfilled.

\section{Appendix C: Equations of motion for the linear perturbations}

As discussed in the main text, the equations of motion for the perturbations are derived from the quadratic action $S_{\text {quad }}\left[h_{\mu \nu}, \delta \phi\right]$ :

$$
\mathcal{E}_{\mu \nu} \equiv \frac{\delta S_{\text {quad }}}{\delta h_{\mu \nu}}=0, \quad \mathcal{E}_{\phi} \equiv \frac{\delta S_{\text {quad }}}{\delta \phi}=0 .
$$

The equation $\mathcal{E}_{\phi}=0$ turns out to be redundant as a consequence of Bianchi's identities, so we just need to take into account the 10 metric equations $\mathcal{E}_{\mu \nu}=0$. Furthermore, due to the spherical symmetry, the equations $\mathcal{E}_{t \varphi}, \mathcal{E}_{r \varphi}$ and $\mathcal{E}_{\varphi \varphi}$ are obviously equivalent to $\mathcal{E}_{t \theta}, \mathcal{E}_{r \theta}$ and $\mathcal{E}_{\theta \theta}$ respectively. Hence, at this stage of the analysis, seven equations at most out of the initial ten equations are independent. We are going to see that we can reduce even more the set of independent equations. This is expected as the number of independent equations must be the same as the number of independent dynamical variables.

\section{Axial perturbations}

The symmetry of the background implies that $\mathcal{E}_{t t}=0, \mathcal{E}_{t r}=0$ and $\mathcal{E}_{r r}=0$. This leaves four non trivial independent equations for two independent functions $h_{0}$ and $h_{1}$. One can thus expect that two of these equations are redundant, which is indeed the case.

First, one has $\mathcal{E}_{\theta \theta}+2 \mathcal{E}_{\theta \varphi}=0$. Then, one can notice that, out of these four equations, $\mathcal{E}_{t \theta}$ contains second-order derivatives of $h_{0}$ and $h_{1}$ while the others contain at most first order derivatives. This is an indication that $\mathcal{E}_{t \theta}$ is redundant and, as expected, one can show that a combination of $\mathcal{E}_{t \theta}$, $\mathcal{E}_{\theta \theta}, \mathcal{E}_{r \theta}$ and their derivatives vanishes. As a consequence, the dynamics of the axial perturbations is fully determined by the system consisting of the two equations

$$
\mathcal{E}_{r \theta}=0, \quad \mathcal{E}_{\theta \theta}=0
$$

for the two variables $h_{0}$ and $h_{1}$. These two equations are first order with respect to the radial coordinate $r$, second order in $\omega$ and are explicitly given in section III A.

\section{Polar perturbations}

Similarly to axial perturbations, we start with seven equations of motion $\mathcal{E}_{\mu \nu}$ but they now depend on five functions: $H_{0}, H_{1}, H_{2}, K$ and $\delta \phi$.

\section{a. BCL black hole perturbations}

In the BCL case, the equation $\mathcal{E}_{\theta \varphi}$ is algebraic, as in GR, and yields $H_{2}$,

$$
H_{2}=\frac{r_{\mathrm{m}}\left(r+\xi r_{\mathrm{m}}\right)}{r^{3}} \delta \phi+\frac{r^{2}+\xi r_{\mathrm{m}}^{2}}{r^{2}} H_{0}
$$


Among the remaining six equations for four independent functions, it turns out that the four equations $\mathcal{E}_{t r}, \mathcal{E}_{r r}, \mathcal{E}_{t \theta}$ and $\mathcal{E}_{r \theta}$ are independent, first-order with respect to the radial coordinate and they imply the last two ones, $\mathcal{E}_{t t}$ and $\mathcal{E}_{\theta \theta}$.

Contrary to GR, the remaining four equations cannot be reduced further because the system now contains two coupled degrees of freedom, the usual polar gravitational mode and the scalar mode. Hence, we obtain a system of four first order equations for the four functions $H_{0}, H_{1}, K$ and $\delta \phi$, whose explicit form is given in (5.6).

\section{b. Stealth black hole perturbations}

We proceed as in the previous case. The equation $\mathcal{E}_{\theta \varphi}$,

$$
\frac{r\left(1+2 q^{2} \alpha\right)-r_{\mathrm{s}}}{r-r_{\mathrm{s}}} H_{0}-4 q^{2} \alpha \frac{\sqrt{r r_{\mathrm{s}}}}{r-r_{\mathrm{s}}} H_{1}-\frac{r-\left(1+2 q^{2} \alpha\right) r_{\mathrm{s}}}{r-r_{\mathrm{s}}} H_{2}-2 q \alpha \sqrt{\frac{r_{\mathrm{s}}}{r^{3}}} \delta \varphi=0,
$$

is algebraic and gives $\mathrm{H}_{2}$ in terms of the other functions. Once again, the four equations $\mathcal{E}_{t r}, \mathcal{E}_{r r}$, $\mathcal{E}_{t \theta}$ and $\mathcal{E}_{r \theta}$ form a complete dynamical system for $H_{0}, H_{1}, K$ and $\delta \phi$. It can be written in the form

$$
M_{A} \frac{\mathrm{d} X}{\mathrm{~d} r}=M_{B} X, \quad X \equiv^{T}\left(\begin{array}{llll}
K & \delta \phi & H_{1} & H_{0}
\end{array}\right),
$$

where the expressions of the matrices $M_{A}$ and $M_{B}$ are quite cumbersome. To simplify, we restrict ourselves to the case where only $\beta \neq 0$ (and $\alpha=\gamma=\delta=0$ ) where $M_{A}$ and $M_{B}$ can be decomposed in powers of $\omega$ according to

$$
M_{A}=M_{A[0]}+M_{A[1]} \omega \quad M_{B}=M_{B[0]}+M_{B[1]} \omega+M_{B[2]} \omega^{2},
$$

with

$$
\begin{aligned}
& M_{A[0]}=\left(\begin{array}{cccc}
\frac{4 \beta q^{4} \sqrt{r_{\mathrm{s}}^{3} r}}{r-r_{\mathrm{s}}} & \frac{16 \beta r_{\mathrm{s}} q^{3}}{r-r_{\mathrm{s}}} & 0 & 8 \beta q^{4} \sqrt{r_{\mathrm{s}} r} \\
-\frac{r\left(r_{\mathrm{s}}^{2}\left(1-4 \beta q^{4}\right)+2 r^{2}-3 r_{\mathrm{s}} r\right)}{\left(r-r_{\mathrm{s}}\right)^{2}} & \frac{16 \beta q^{3} \sqrt{r_{\mathrm{s}}^{3} r}}{\left(r-r_{\mathrm{s}}^{2}\right.} & 0 & \frac{2 r\left(r_{\mathrm{s}}\left(4 \beta q^{4}-1\right)+r\right)}{r-r_{\mathrm{s}}} \\
0 & -\frac{4 \beta r_{\mathrm{s}} q^{3}}{r} & r_{\mathrm{s}}-r & 0 \\
r & -\frac{4 \beta r_{\mathrm{s}}^{3 / 2} q^{3}}{\sqrt{r}\left(r-r_{\mathrm{s}}\right)} & 0 & -r
\end{array}\right), \\
& M_{A[1]}=\left(\begin{array}{cccc}
-2 i r^{2} & -16 i \beta q^{3} \sqrt{r_{\mathrm{s}} r} & 0 & 0 \\
0 & 0 & 0 & 0 \\
0 & 0 & 0 & 0 \\
0 & 0 & 0 & 0
\end{array}\right) \text {, } \\
& M_{B[0]}=\left(\begin{array}{cccc}
0 & -\frac{8 \beta(\lambda+1) r_{\mathrm{s}} q^{3}}{r\left(r-r_{\mathrm{s}}\right)} & -\frac{2\left((\lambda+1) r_{\mathrm{s}}^{2}-2 r_{\mathrm{s}} r\left(\lambda+4 \beta q^{4}+1\right)+(\lambda+1) r^{2}\right)}{\left(r-r_{\mathrm{s}}\right)^{2}} & -\frac{16 \beta q^{4} \sqrt{r_{\mathrm{s}}^{3} r}}{\left(r-r_{\mathrm{s}}\right)^{2}} \\
\frac{2 \lambda r}{r-r_{\mathrm{s}}} & -\frac{8 \beta(\lambda+1) r_{\mathrm{s}}^{3 / 2} q^{3}}{\sqrt{r}\left(r-r_{\mathrm{s}}\right)^{2}} & \frac{16 \beta q^{4}\left(r_{\mathrm{s}} r\right)^{3 / 2}}{\left(r-r_{\mathrm{s}}\right)^{3}} & -\frac{2 r\left(r_{\mathrm{s}}^{2}\left(\lambda+8 \beta q^{4}\right)+\lambda r^{2}-2 \lambda r_{\mathrm{s}} r\right)}{\left(r-r_{\mathrm{s}}\right)^{3}} \\
0 & 0 & -\frac{r_{\mathrm{s}}\left(-r_{\mathrm{s}}+4 \beta q^{4} r+r\right)}{r\left(r-r_{\mathrm{s}}\right)} & \frac{2 \beta q^{4} \sqrt{\frac{r_{\mathrm{s}}}{r}}\left(r_{\mathrm{s}}+r\right)}{r-r_{\mathrm{s}}} \\
0 & 0 & -\frac{4 \beta q^{4} \sqrt{r_{\mathrm{s}} r}}{\left(r-r_{\mathrm{s}}\right)^{2}} & \frac{r_{\mathrm{s}}\left(r_{\mathrm{s}}+2 \beta r_{\mathrm{s}} q^{4}+r\left(2 \beta q^{4}-1\right)\right)}{\left(r-r_{\mathrm{s}}\right)^{2}}
\end{array}\right),
\end{aligned}
$$




$$
\begin{gathered}
M_{B[1]}=\left(\begin{array}{cccc}
-\frac{i r\left(3 r_{\mathrm{s}}^{2}-r_{\mathrm{s}} r\left(4 \beta q^{4}+5\right)+2 r^{2}\right)}{\left(r-r_{\mathrm{s}}\right)^{2}} & \frac{16 i \beta q^{3} \sqrt{r_{\mathrm{s}}^{3} r}}{\left(r-r_{\mathrm{s}}\right)^{2}} & 0 & \frac{2 i r\left(r_{\mathrm{s}}\left(4 \beta q^{4}-1\right)+r\right)}{r-r_{\mathrm{s}}} \\
\frac{4 i \beta r_{\mathrm{s}}^{3 / 2} q^{4} r^{5 / 2}}{\left(r-r_{\mathrm{s}}\right)^{3}} & \frac{16 i \beta r_{\mathrm{s}}^{2} q^{3} r}{\left(r-r_{\mathrm{s}}\right)^{3}} & \frac{4 i r^{2}\left(r_{\mathrm{s}}\left(4 \beta q^{4}-1\right)+r\right)}{\left(r-r_{\mathrm{s}}\right)^{2}} & -\frac{8 i \beta q^{4} \sqrt{r_{\mathrm{s}} r^{5}}}{\left(r-r_{\mathrm{s}}\right)^{2}} \\
-i r & -\frac{4 i \beta q^{3} \sqrt{r_{\mathrm{s}} r}}{r-r_{\mathrm{s}}} & 0 & -i r \\
0 & -\frac{4 i \beta r_{\mathrm{s}} q^{3} r}{\left(r-r_{\mathrm{s}}\right)^{2}} & -\frac{i r^{2}}{r-r_{\mathrm{s}}} & 0
\end{array}\right), \\
M_{B[2]}=\left(\begin{array}{ccccc}
0 & 0 & 0 & 0 \\
-\frac{2 r^{4}}{\left(r-r_{\mathrm{s}}\right)^{2}} & -\frac{16 \beta q^{3} \sqrt{r_{\mathrm{s}} r^{5}}}{\left(r-r_{\mathrm{s}}\right)^{2}} & 0 & 0 \\
0 & 0 & 0 & 0 \\
0 & 0 & 0 & 0
\end{array}\right) .
\end{gathered}
$$

We do not write down the general equations (i.e. with generic values for $\alpha, \beta, \gamma$ and $\delta$ ) which are particularly cumbersome.

\section{Appendix D: Schrödinger-like equation from a general two-dimensional system}

In this Appendix, we consider a two-dimensional first-order differential system of the form,

$$
\frac{\mathrm{d} Y}{\mathrm{~d} r}=M Y, \quad M(r)=M_{[0]}(r)+\omega M_{[1]}(r)+\omega^{2} M_{[2]}(r),
$$

where the matrices $M_{[0]}, M_{[1]}$ and $M_{[2]}$ do not depend on $\omega$. Their coefficients, which are functions of $r$, will be denoted $a_{n}, b_{n}, c_{n}$ and $d_{n}$, so that

$$
M_{[n]}(r)=\left(\begin{array}{ll}
a_{n}(r) & b_{n}(r) \\
c_{n}(r) & d_{n}(r)
\end{array}\right)
$$

The system admits a Schrödinger-like form if one can find a new vector $\hat{Y}$ related to $Y$ by the transformation $Y=\hat{P} \hat{Y}$, where the transition matrix $\hat{P}$ depends on $r$ but not on $\omega$, leading to a system of the form

$$
\frac{\mathrm{d} \hat{Y}}{\mathrm{~d} r}=\hat{M} \hat{Y}, \quad \text { with } \quad \hat{M}(r)=\frac{1}{n(r)}\left(\begin{array}{cc}
i \omega \mu(r) & 1 \\
V(r)-\omega^{2} / c^{2}(r) & i \omega \mu(r)
\end{array}\right),
$$

where $n, \mu, V$ and $c$ are functions of $r$. In particular, $n(r)$ allows for a possible rescaling of the radial coordinate.

Using similar notations as in (D1), we can decompose $\hat{M}$ as

$$
\hat{M}(r)=\hat{M}_{[0]}(r)+\omega \hat{M}_{[1]}(r)+\omega^{2} \hat{M}_{[2]}(r),
$$

where the invidual matrices can be read off from (D3) and are related to the matrices in (D1) by

$$
\hat{M}_{[2]}=\hat{P}^{-1} M_{[2]} \hat{P}, \quad \hat{M}_{[1]}=\hat{P}^{-1} M_{[1]} \hat{P}, \quad \hat{M}_{[0]}=\hat{P}^{-1} M_{[0]} \hat{P}^{-} \hat{P}^{-1} \hat{P}^{\prime},
$$

where $\hat{P}^{\prime}$ denotes the derivative of $\hat{P}$ with respect to $r$. One notices from (D3) that $\hat{M}_{[1]}$ is proportional to the identity matrix and $\hat{M}_{[2]}$ is nilpotent. Given (D5), this requires that the original matrices $M_{[1]}$ and $M_{[2]}$ satisfy the same properties, respectively. This implies in particular that $\hat{M}_{[1]}=M_{[1]}$ and therefore

$$
\frac{\mu}{n}=a_{1}=d_{1}
$$


In the following, we will assume, for simplicity, that

$$
M_{[2]}=\left(\begin{array}{cc}
0 & 0 \\
c_{2} & 0
\end{array}\right)
$$

Indeed, since $M_{[2]}$ is nilpotent, it is always possible to make a transformation $X=\tilde{P} \tilde{X}$ to bring the matrix coefficient of $\omega^{2}$ in this form, so there is no loss of generality with the above assumption. It is then easy to check, using the first relation in (D5), that the most general $\hat{P}$ that brings $M_{[2]}$ of the form D7) into $\hat{M}_{[2]}$ corresponding to (D3) is

$$
\hat{P}=x\left(\begin{array}{ll}
1 & 0 \\
y & z
\end{array}\right) \quad \text { with } \quad z=-c^{2} n c_{2},
$$

where $y$ and $x$ are arbitrary (and $x \neq 0$ ).

The functions $x$ and $y$ can be determined by requesting that the initial matrix $M_{[0]}$ is transformed into the requested form $\hat{M}_{[0]}$. Using the third transformation relation in (D5), this leads to the four equations

$$
\begin{aligned}
& x^{\prime}-\left(a_{0}+y b_{0}\right) x=0, \\
& 1-b_{0} n z=0, \\
& (x y)^{\prime}-\left(c_{0}+y d_{0}\right) x+V \frac{x z}{n}=0, \\
& (x z)^{\prime}-d_{0} x z+\frac{x y}{n}=0 .
\end{aligned}
$$

The second equation, Eq. (D10), is purely algebraic and is solved by

$$
z=\frac{1}{n b_{0}},
$$

which can be substituted into both (D11) and (D12). The combination of (D9) and (D12) then yields

$$
x=\sqrt{b_{0} n} \exp \frac{1}{2}\left[\int^{r} \mathrm{~d} u\left(a_{0}(u)+d_{0}(u)\right)\right], \quad y=\frac{1}{2 b_{0}}\left(d_{0}-a_{0}+\frac{b_{0}^{\prime}}{b_{0}}+\frac{n^{\prime}}{n}\right),
$$

and, finally, the expression of the potential follows from (D11),

$$
V=n^{2} b_{0}\left(c_{0}+y d_{0}-y \frac{x^{\prime}}{x}-y^{\prime}\right) \text {. }
$$

Substituting the solutions (D14) for $x$ and $y$, we obtain the simple expression

$$
V=\frac{n^{2}}{4}\left[4 b_{0} c_{0}+\left(d_{0}-a_{0}\right)^{2}-2\left(d_{0}^{\prime}-a_{0}^{\prime}\right)+2 \frac{b_{0}^{\prime}}{b_{0}}\left(d_{0}-a_{0}\right)+3\left(\frac{b_{0}^{\prime}}{b_{0}}\right)^{2}+\left(\frac{n^{\prime}}{n}\right)^{2}-2\left(\frac{b_{0}^{\prime \prime}}{b_{0}}+\frac{n^{\prime \prime}}{n}\right)\right] .
$$

This potential, valid for an arbitrary choice of radial coordinate, i.e. of $n$, is associated with the propagation speed

$$
c^{2}=-\frac{1}{n^{2} b_{0} c_{2}}
$$

obtained from (D8) and (D13). 
In conclusion, for any differential system of the form (D1), we have found the necessary and sufficient conditions for it to be rewritten in a Schrödinger-like form: $M_{[1]}$ must be proportional to the identity matrix and $M_{[2]}$ nilpotent. In this case, and assuming the form (D7) for the matrix $M_{[2]}$ we have obtained explicitly the potential $V$ and the propagation speed $c$, given respectively by (D16) and (D17).

Let us apply the above results to the system (3.7) for axial perturbations. One must first transform the system so that the matrix coefficient of $\omega^{2}$ has the canonical form (D7). This can be done via the transformation $Y=\tilde{P} \tilde{Y}$ with

$$
\tilde{P}=\left(\begin{array}{ll}
0 & 1 \\
1 & 0
\end{array}\right), \quad \tilde{c}_{2}=b_{2}
$$

so that the new (non vanishing) coefficients are

$$
\begin{aligned}
& \tilde{a}_{0}=d_{0}=\Delta, \quad \tilde{b}_{0}=c_{0}=-i \Gamma, \quad \tilde{c}_{0}=b_{0}=2 i \lambda \frac{\Phi}{r^{2}}, \quad \tilde{d}_{0}=a_{0}=\frac{2}{r}, \\
& \tilde{a}_{1}=\tilde{d}_{1}=a_{1}=i \Psi, \quad \tilde{c}_{2}=b_{2}=-i .
\end{aligned}
$$

Using the expressions (D17) and (D16) with the "tilded" coefficients we obtain, respectively,

$$
c^{2}=\frac{1}{n^{2} \Gamma}
$$

and

$$
V=\frac{n^{2}}{4}\left[\frac{8(1+\lambda \Phi \Gamma)}{r^{2}}+\Delta^{2}-\frac{4 \Delta}{r}+2 \Delta^{\prime}+\frac{2 \Gamma^{\prime}}{\Gamma}\left(\frac{2}{r}-\Delta\right)+3\left(\frac{\Gamma^{\prime}}{\Gamma}\right)^{2}+\left(\frac{n^{\prime}}{n}\right)^{2}-2\left(\frac{\Gamma^{\prime \prime}}{\Gamma}+\frac{n^{\prime \prime}}{n}\right)\right] .
$$

The radial rescaling is arbitrary and one can choose it so that the propagation speed is normalised, i.e. $c=1$. According to (D21), this corresponds to the choice

$$
n_{c=1}=\frac{1}{\sqrt{\Gamma}} .
$$

Substituting in the general expression, the potential becomes

$$
V_{c=1}=\frac{1}{4 \Gamma}\left[\frac{8(1+\lambda \Phi \Gamma)}{r^{2}}+\Delta^{2}-\frac{4 \Delta}{r}+2 \Delta^{\prime}+\frac{2 \Gamma^{\prime}}{\Gamma}\left(\frac{2}{r}-\Delta\right)+\frac{7 \Gamma^{\prime 2}}{4 \Gamma^{2}}-\frac{\Gamma^{\prime \prime}}{\Gamma}\right] .
$$

This expression can be applied in particular to the first-order system governing polar perturbations about a Schwarzschild black hole in GR, as recalled in paper I. In this case, one recovers the usual Regge-Wheeler potential.

\section{Appendix E: Details on the BCL black hole perturbations}

In this Appendix, we give more details on the asymptotic analysis of polar perturbations about the BCL solution. 


\section{At spatial infinity}

The final variable $\tilde{Y}$ (which diagonalises the dynamical system up to the order $1 / r^{2}$ at spatial infinity) is related to the original variable $Y$ by the linear transformation $Y=\tilde{P} \tilde{Y}$ with

$$
\tilde{P}=\left(\begin{array}{cccc}
p_{1}+q_{1} & p_{1}-q_{1} & r_{1}+s_{1} & r_{1}-s_{1} \\
0 & 0 & r_{2}+s_{2} & r_{2}-s_{2} \\
p_{3}+q_{3} & p_{3}-q_{3} & r_{3}+s_{3} & r_{3}-s_{3} \\
p_{3}+q_{3} & -p_{3}+q_{3} & r_{4}+s_{4} & r_{4}-s_{4}
\end{array}\right)
$$

where the coefficients are given by

$$
\begin{aligned}
& p_{1}=-\frac{2 \lambda}{3 r \omega^{2}}, \quad q_{1}=\frac{i\left(3 r-2 r_{\mathrm{s}}\right)}{3 r \omega}, \quad r_{1}=\frac{27-10 \lambda}{12 r \omega^{2}}, \quad s_{1}=-\frac{\sqrt{2}\left(12 r+7 r_{\mathrm{s}}\right)}{24 r \omega}, \\
& r_{2}=\frac{\sqrt{2}(3-2 \lambda) r}{8 \omega}, \quad s_{2}=\frac{\left(12 r+7 r_{\mathrm{s}}\right) r}{8}, \quad p_{3}=\frac{3 r+r_{\mathrm{s}}}{3}, \quad q_{3}=-\frac{i \lambda}{3 \omega}, \\
& r_{3}=\frac{i(2 \lambda-9)}{6 \omega}, \quad s_{3}=\frac{i \sqrt{2}\left(11 r_{\mathrm{s}}-12 r\right)}{12}, \quad r_{4}=\frac{12 r-5 r_{\mathrm{s}}}{12}, \quad s_{4}=-\frac{\sqrt{2}(27+2 \lambda)}{12 \omega} .
\end{aligned}
$$

As we announced, this change of variable enables us to diagonalise the system whose associated matrix $\tilde{M}$ is

$$
\tilde{M}(r)=\omega\left(\begin{array}{cccc}
-i & 0 & 0 & 0 \\
0 & i & 0 & 0 \\
0 & 0 & -\sqrt{2} & 0 \\
0 & 0 & 0 & \sqrt{2}
\end{array}\right)+\frac{1}{r}\left(\begin{array}{cccc}
-i \omega r_{\mathrm{m}} & 0 & 0 & 0 \\
0 & i \omega r_{\mathrm{m}} & 0 & 0 \\
0 & 0 & -3-\frac{\omega r_{\mathrm{m}}}{\sqrt{2}} & 0 \\
0 & 0 & 0 & -3+\frac{\omega r_{\mathrm{m}}}{\sqrt{2}}
\end{array}\right)+\mathcal{O}\left(\frac{1}{r^{2}}\right)
$$

up to the order $\mathcal{O}\left(1 / r^{2}\right)$. One can easily check that the dominant term in the asymptotic expansion of $\tilde{M}$ is a diagonalisation of $M_{0}^{(1)}(5.12)$ as expected.

\section{Near the horizon}

As we showed in section VA2, we can make a first change of variables

$$
Y=P_{(1)} Y^{(1)}, \quad P_{(1)}=\left(\begin{array}{cccc}
1 & 0 & 0 & 0 \\
0 & 1 / \varepsilon & 0 & 0 \\
0 & 0 & 1 / \varepsilon & 0 \\
0 & 0 & 0 & 1 / \varepsilon
\end{array}\right)
$$

so that the new differential system satisfied by $Y^{(1)}$ has an associated matrix $M^{(1)}$ with a very simple asymptotic expansion,

$$
M^{(1)}=\frac{1}{\varepsilon} M_{0}^{(1)}+\mathcal{O}(1)
$$

where the matrix $M_{0}^{(1)}$ is given by,

$$
M_{0}^{(1)}=\left(\begin{array}{cccc}
\frac{1}{2} & \frac{\eta}{r_{0} r_{+}} & i \frac{1+\lambda}{\omega r_{+}^{2}} & \frac{\eta^{2}}{r_{+}} \\
\frac{r_{+}^{2}}{4}+\frac{\omega^{2} r_{0}^{2} r_{+}^{2}}{\eta^{2}} & \frac{\eta^{2}}{2} & i \frac{1+\lambda}{2 \omega}-\frac{i \omega r_{+} r_{0}}{\eta} & \frac{5-\eta^{2}+2 \eta^{4}+4 \lambda}{4 \eta} r_{0} \\
0 & -i \omega & 0 & -i \eta \omega r_{0} \\
0 & -\frac{\eta}{2 r_{0}} & -\frac{i \omega r_{0}}{\eta} & \frac{1-\eta^{2}}{2} .
\end{array}\right) .
$$


Even though the expression of $M^{(1)}$ is relatively complex, it can be transformed into a simple Jordan block form with two Jordan blocks. Indeed, we make a new change of variable $Y^{(1)}=P_{(2)} Y^{(2)}$ where $P_{(2)}$ transforms $M_{0}^{(1)}$ according to

$$
M_{0}^{(1)}=P_{(2)}\left(\begin{array}{cccc}
-i \omega r_{0} & 0 & 0 & 0 \\
0 & +i \omega r_{0} & 0 & 0 \\
0 & 0 & 1 / 2 & 1 \\
0 & 0 & 0 & 1 / 2
\end{array}\right) P_{(2)}^{-1}
$$

The solution for $Y^{(2)}$ is obtained immediately and reads

$$
Y^{(2)}(r)=\left(\begin{array}{c}
c_{-} \varepsilon^{-i \omega r_{0}} \\
c_{+} \varepsilon^{+i \omega r_{0}} \\
\left(a_{1} \ln \varepsilon+a_{2}\right) \sqrt{\varepsilon} \\
a_{1} \sqrt{\varepsilon}
\end{array}\right)(1+\mathcal{O}(\varepsilon)),
$$

where $c_{ \pm}, a_{1}$ and $a_{2}$ are constant.

The asymptotic expansion at the horizon of the original variable $Y$ whose components are the metric and scalar perturbations $(5.6)$ is obtained directly from the matrix of change of variables $P$ such that $Y=P Y^{(2)}$. It is given by the product $P=P_{(1)} P_{(2)}$ which reads after a direct calculation,

$$
P=\frac{1}{\varepsilon}\left(\begin{array}{cccc}
-\frac{2 \rho \varepsilon\left(i \eta r_{+} \omega+1+\lambda\right)}{\omega r_{+}^{3 / 2} \Delta_{1}} & \frac{2 \rho \varepsilon\left(i \eta r_{+} \omega-1-\lambda\right)}{\omega r_{+}^{3 / 2} \Delta_{2}} & -\frac{2 \rho \varepsilon\left((3+2 \lambda) r_{+}+r_{-}\right)}{r_{+} \Delta_{3}} & i \varepsilon \frac{4\left(2 r_{+}+3 r_{-}\right) r_{+}^{3} \omega^{2}-(1+\lambda) \rho^{2}}{r_{+}^{2} \Delta_{3}} \\
-\frac{2 i \eta r_{-} r_{+}^{3 / 2}}{\Delta_{1}} & \frac{2 i \eta r_{-} r_{+}^{3 / 2}}{\Delta_{2}} & -\frac{r_{+}\left(r_{+}+2 r_{-}\right)}{\rho} & \frac{i}{2 \omega} \\
-\frac{i r_{+}^{1 / 2}\left(\rho+2 i \eta r_{+}^{2} \omega\right)}{\Delta_{1}} & -\frac{i r_{+}^{1 / 2}\left(\rho+2 i \eta r_{+}^{2} \omega\right)}{\Delta_{2}} & 0 & 1 \\
1 & 1 & 1 & 0
\end{array}\right)
$$

where we introduced the notations $\rho \equiv r_{+}+r_{-}$and

$$
\Delta_{1} \equiv \sqrt{r_{+}}\left(2 \omega r_{+}^{2}+i \eta \rho\right), \quad \Delta_{2} \equiv \sqrt{r_{+}}\left(2 \omega r_{+}^{2}-i \eta \rho\right), \quad \Delta_{3} \equiv \rho^{2}+4 \omega^{2} r_{+}^{4} .
$$

\section{Appendix F: Polar perturbations about the stealth solution: the K-essence case}

In this appendix, we study the polar perturbations of the stealth solution in Horndeski theory where the only non-vanishing parameter among (2.9) is $\gamma \neq 0$. In this case, the action (2.1) reduces to the sum of the Einstein-Hilbert term supplemented with a so-called K-essence term and simply reads

$$
S\left[\phi, g_{\mu \nu}\right]=\int d^{4} x \sqrt{-g}\left(R+\frac{\gamma}{2}\left(X+q^{2}\right)^{2}\right) .
$$

Following the notations and the procedure we described in the paper, we can compute the corresponding polar perturbations equations about the stealth solution. As expected, they can be cast into a form very similar to those of GR, with three first order equations

$$
\begin{aligned}
& K^{\prime}-\frac{1}{r} H_{0}-\frac{i(\lambda+1)}{r^{2} \omega} H_{1}+\frac{2 r-3 r_{\mathrm{s}}}{2 r\left(r-r_{\mathrm{s}}\right)} K=\frac{i q^{2} \gamma \sqrt{r r_{\mathrm{s}}}}{\omega\left(r-r_{\mathrm{s}}\right)} \delta X, \\
& H_{1}^{\prime}+\frac{i r \omega}{r-r_{\mathrm{s}}} H_{0}+\frac{r_{\mathrm{s}}}{r\left(r-r_{\mathrm{s}}\right)} H_{1}+\frac{i r \omega}{r-r_{\mathrm{s}}} K=0, \\
& H_{0}^{\prime}-K^{\prime}+\frac{r_{\mathrm{s}}}{r\left(r-r_{\mathrm{s}}\right)} H_{0}+\frac{i r \omega}{r-r_{\mathrm{s}}} H_{1}=0 .
\end{aligned}
$$


along with one algebraic relation,

$$
\begin{aligned}
0 & =\left(2 \lambda+\frac{3 r_{\mathrm{s}}}{r}\right) H_{0}+\left(\frac{i(\lambda+1) r_{\mathrm{s}}}{r^{2} \omega}-2 i r \omega\right) H_{1} \\
& +\left(-\frac{4 r \lambda\left(r-r_{\mathrm{s}}\right)+2 r r_{\mathrm{s}}-3 r_{\mathrm{s}}^{2}}{2 r\left(r-r_{\mathrm{s}}\right)}+\frac{2 r^{3} \omega^{2}}{r-r_{\mathrm{s}}}\right) K+\left(\frac{2 q^{2} \gamma r^{2} r_{\mathrm{s}}}{r-r_{\mathrm{s}}}+\frac{i q^{2} \gamma \sqrt{r r_{\mathrm{s}}} r_{\mathrm{s}}}{\omega\left(r-r_{\mathrm{s}}\right)}\right) \delta X
\end{aligned},
$$

where we have chosen to keep explicitly $\delta X$, the linear perturbation of $X=\phi_{\mu} \phi^{\mu} . \delta X$ can also be expressed in terms of $\delta \phi, H_{0}, H_{1}$ and $K$ :

$$
\delta X=-\frac{q^{2}\left(r_{\mathrm{s}}+r\right)}{r-r_{\mathrm{s}}} H_{0}+\frac{2 q^{2} \sqrt{r_{\mathrm{s}} r}}{r-r_{\mathrm{s}}} H_{1}+2 q \sqrt{\frac{r_{\mathrm{s}}}{r}} \delta \phi^{\prime}+\frac{2 i q r \omega}{r-r_{\mathrm{s}}} \delta \phi .
$$

At this stage, it is possible to treat the system $(\sqrt{\mathrm{F} 2})$ and $(\mathrm{F} 3)$ in the same way we have treated the system for polar perturbations in GR (see Paper I). We first solve the algebraic equation (F3) for $H_{0}$ and then substitute the solution into the first two differential equations (F2). Hence, we obtain a system of the form

$$
\frac{\mathrm{d} Y}{\mathrm{~d} r}-M(r) Y=\frac{q^{2} \gamma \delta X}{\left(r-r_{\mathrm{s}}\right)\left(2 r \lambda+3 r_{\mathrm{s}}\right)}\left(\begin{array}{c}
2 r^{2} r_{\mathrm{s}}-2 i \sqrt{r r_{\mathrm{s}}}\left(r \lambda+r_{\mathrm{s}}\right) / \omega \\
r_{\mathrm{s}} r^{2} \sqrt{r r_{\mathrm{s}}} /\left(r-r_{\mathrm{s}}\right)-2 i r^{4} r_{\mathrm{s}} \omega /\left(r-r_{\mathrm{s}}\right)
\end{array}\right),
$$

where $Y \equiv{ }^{T}\left(K H_{1}\right)$ and $M(r)$ is the matrix entering in the dynamical system of polar perturbations in GR whose expression has been computed in the companion paper,

$$
M(r)=\frac{1}{3 r_{\mathrm{s}}+2 \lambda r}\left(\begin{array}{cc}
\frac{r_{\mathrm{s}}\left(3 r_{\mathrm{s}}+(\lambda-2) r\right)-2 r^{4} \omega^{2}}{r\left(r-r_{\mathrm{s}}\right)} & \frac{2 i(\lambda+1)\left(r_{\mathrm{s}}+\lambda r\right)+2 i r^{3} \omega^{2}}{r^{2}} \\
\frac{i r\left(9 r_{\mathrm{s}}^{2}-8 \lambda r^{2}+8(\lambda-1) r_{\mathrm{s}} r\right)+4 i r^{5} \omega^{2}}{2\left(r-r_{\mathrm{s}}\right)^{2}} & \frac{2 r^{4} \omega^{2}-r_{\mathrm{s}}\left(3 r_{\mathrm{s}}+3 \lambda r+r\right)}{r\left(r-r_{\mathrm{s}}\right)}
\end{array}\right) .
$$

We can therefore interpret the system (F5) as describing the dynamics of unmodified polar perturbations in GR on which the scalar field acts like a source.

Finally, it is possible to obtain a fully decoupled equation for the perturbation $\delta X$. For that one replaces the expressions of $H_{0}^{\prime}, H_{1}^{\prime}$ and $K^{\prime}$ (computed from (F5) or the algebraic equation) into (F2). After a direct calculation, one obtains

$$
i r^{2}\left(\sqrt{r r_{\mathrm{s}}}-2 i r^{2} \omega\right) \delta X^{\prime}(r)+\left(\frac{3}{2} i r \sqrt{r r_{\mathrm{s}}}+r^{3}\left(3-\frac{r}{r-r_{\mathrm{s}}}\right) \omega+\frac{2 i r^{5}}{r-r_{\mathrm{s}}} \sqrt{\frac{r}{r_{\mathrm{s}}}} \omega^{2}\right) \delta X(r)=0,
$$

which, after some simplifications, becomes $\$ 15$

$$
2 \sqrt{r_{\mathrm{s}}}\left(r-r_{s}\right) r \delta X^{\prime}(r)+\sqrt{r_{s}}\left(3\left(r-r_{s}\right)+2 i r^{2} \sqrt{r / r_{s}}\right) \delta X(r)=0 .
$$

The equation for $\delta X(r)$ can be solved explicitly and one finds

$$
\delta X(r)=\frac{C}{r^{3 / 2}}\left(\frac{\sqrt{r}+\sqrt{r_{\mathrm{s}}}}{\sqrt{r}-\sqrt{r_{\mathrm{s}}}}\right)^{i \omega r_{\mathrm{s}}} \exp \left(-\frac{2}{3} i \omega\left(r+3 r_{\mathrm{s}}\right) \sqrt{r / r_{\mathrm{s}}}\right),
$$

where $C$ is an integration constant. Hence, the asymptotics of $\delta X$ are deduced immediately and one obtains,

$$
\delta X(r) \approx \frac{C}{z^{3}} \exp \left(-2 i \omega z r_{\mathrm{s}}\left(z^{2} / 3+1\right)\right)(1+\mathcal{O}(1 / z)), \quad z \equiv \sqrt{r / r_{\mathrm{s}}} \gg 1
$$

15 Notice that such a decoupled equation for $\delta X$ was expected. Indeed, we can directly check that it is exactly the same as the well-known conservation equation (for linear perturbations) in shift-symmetric theories,

$$
\nabla_{\mu}\left(\sqrt{-g} \delta X e^{-i \omega t} \phi^{\mu}\right)=\frac{1}{\sqrt{-g}} \partial_{\mu}\left(\sqrt{-g} \delta X e^{-i \omega t} \phi^{\mu}\right)=0 .
$$


at infinity, and

$$
\delta X(r) \approx D\left(r-r_{\mathrm{s}}\right)^{-i \omega r_{\mathrm{s}}}\left(1+\mathcal{O}\left(r-r_{\mathrm{s}}\right)\right), \quad r-r_{\mathrm{s}} \ll r_{\mathrm{s}}
$$

near the horizon, where $D$ is a constant that can be computed trivially.

In order to compute the asymptotic behavior of $\delta \varphi$, we need to solve (F4). But, at this stage, it is already remarkable to observe that the asymptotic behaviour of $\delta X$ agrees with the asymptotic behaviour of $\delta \phi$ computed in (5.25) and (5.26) from the first order system.

But, for completeness, let us consider $(\overrightarrow{\mathrm{F}} 4)$ which can be viewed as a first order equation for $\delta \phi$ with three sources proportional to $H_{0}, H_{1}$ and $\delta X$. The first two can be computed from (F5) and the algebraic equation while the third one has just been computed above. By superposition, the general solution is a combination of three particular solutions (solutions where only one of the three sources is turned on) and one homogeneous solution.

The homogeneous equation is

$$
\delta \phi^{\prime}(r)+\sqrt{\frac{r}{r_{\mathrm{s}}}} \frac{i \omega r}{r-r_{\mathrm{s}}} \delta \phi(r)=0 .
$$

It can be fully integrated, and the solution is

$$
\delta \phi=C\left(\frac{z+1}{z-1}\right)^{i \omega r_{\mathrm{s}}} \exp \left(-2 i \omega z r_{\mathrm{s}}\left(z^{2} / 3+1\right)\right), \quad z \equiv \sqrt{r / r_{\mathrm{s}}},
$$

where $C$ is also a constant. We observe that the solution of the homogeneous solution for $\delta \phi$ is almost the same as the solution for $\delta X$. They only differ by the overall factor $r^{3 / 2}$. Hence, their behaviors at infinity and at the horizon are exactly the same ones (up to some integers powers of $z$ that play no role). This means that the homogeneous solution and the particular solution associated with $\delta X$ have the same asymptotics. Moreover, the functions $H_{0}$ and $H_{1}$ have their asymptotic behavior fixed by the modified GR system ( $\overline{\mathrm{F} 5}$ ): they both behave like GR metric modes at infinity and at the horizon.

As a conclusion, $\delta \phi$ can have two different behaviors at infinity and at the horizon (or any linear combination of these two): it can either behave exactly like a metric mode, similarly to $H_{0}$ and $H_{1}$; or it can have the behaviour of $\delta X$ computed previously.

These behaviours are exactly the ones found for the decoupled modes (5.26). We understand now why the branches $n_{+}$and $n_{-}$were the same : the asymptotic scalar behaviour is set by $\delta X$, and $\delta X$ does not verify a second-order equation but a first-order one. A similar behaviour was found for the theory where $\alpha \neq 0$, which means that such a simplification of the equations may also exist in that case.

\section{Appendix G: Linear perturbations of the scalar field about a fixed background in Horndeski theories}

We consider a background solution, for the (static and spherically symmetric) metric $\bar{g}_{\mu \nu}(r)$ and the scalar field $\bar{\phi}(r, t)=q t+\psi(r)$, in Horndeski theories and we study the dynamics of the linear perturbations of the scalar field only $\delta \phi \equiv \phi-\bar{\phi}$ about such a background. Hence, we do not consider perturbations of the metric. As usual, we decompose the perturbation of the scalar field onto spherical harmonics

$$
\delta \phi=\sum_{\ell, m} \delta \phi^{\ell m}(t, r) Y_{\ell m}(\theta, \varphi)
$$


and we study independently each components $\delta \phi^{\ell m}(t, r)$. As these components do not couple at the linear order, we drop the indices $\ell, m$. Then, we consider the Fourier components of $\delta \phi$ which is equivalent to taking $\delta \phi(r, t)=\delta \phi(r) e^{-i \omega t}$ as we have done all along the paper.

One can compute the equation satisfied by $\delta \phi(r)$ in any such background but its general expression is too cumbersome to be written here. In the case $q=0$, it can be extracted from the quadratic Lagrangian computed in [32]. Instead, we concentrate on the two background solutions we have considered in the paper, namely the BCL and the stealth Schwarzschild solutions.

\section{BCL background}

When the background is the BCL metric, one shows that the differential equation satisfied by $\chi(t, r)$ (defined from $\delta \phi(t, r)$ in $(5.5)$ ) is given by

$$
\frac{\partial^{2} \chi}{\partial t^{2}}+\frac{1}{2} A(r) \frac{\partial^{2} \chi}{\partial r^{2}}+\frac{1}{r}\left(1+\frac{r_{\mathrm{m}}^{2} \xi}{2 r^{2}}\right) \frac{\partial \chi}{\partial r}-W(r) \chi=0,
$$

where $A(r)$ is the function entering into the BCL metric and

$$
W(r)=\frac{1}{4 r^{4}}\left(2 r^{2}(3+2 \lambda)-4 r(1+\lambda) r_{\mathrm{m}}-2(1+2 \lambda) r_{\mathrm{m}}^{2} \xi-\frac{1}{2} \frac{\left(2 r-r_{\mathrm{m}}\right)^{2}}{A(r)}\right) .
$$

As $A(r)>0$, one immediately sees that $\chi(r, t)$ satisfies an elliptic equation and is therefore not propagating.

We now consider the Fourier component of $\chi(t, r)$, namely $\chi(r)$, and change variables by writing

$$
\chi(r)=\varpi(r) \tilde{\chi}(r) .
$$

By setting

$$
\varpi(r)=\frac{1}{2 r A(r)},
$$

we obtain the following differential equation for $\tilde{\chi}$ :

$$
\frac{1}{2} A(r) \tilde{\chi}^{\prime \prime}+\left(\frac{r_{\mathrm{m}}^{2} \xi}{2 r^{4}}-W(r)-\omega^{2}\right) \tilde{\chi}=0
$$

When $r \longrightarrow+\infty$, this equation simplifies to

$$
\tilde{\chi}^{\prime \prime}=2 \omega^{2} \tilde{\chi},
$$

which means that the behaviour at infinity of $\chi(r)$ is given by

$$
\chi(r)=\frac{1}{2 r}\left(b_{1} e^{\sqrt{2} \omega r}+b_{2} e^{-\sqrt{2} \omega r}\right),
$$

where $b_{1}$ and $b_{2}$ are integration constants. This agrees with the asymptotic behaviour found for the scalar mode in (5.15). Therefore, it seems that the asymptotic behaviour of the scalar perturbation when the metric is fixed coincides with the asymptotic behavior of the scalar part of the polar modes.

In order to confirm this intuition, we study G5 when $r \longrightarrow r_{+}$. The resulting equation is

$$
\tilde{\chi}^{\prime \prime}+\frac{1}{4\left(r-r_{+}\right)^{2}} \tilde{\chi}=0
$$


and the general solution corresponds to

$$
\chi(r)=\frac{1}{\sqrt{r-r_{+}}}\left(b_{1}+b_{2} \ln \left(r-r_{+}\right)\right),
$$

where $b_{1}$ and $b_{2}$ are integration constants. We observe that this result is also fully consistent with the asymptotic analysis in (E8).

\section{Stealth background}

A similar analysis can be make when the background is the stealth Schwarzschild solution. For simplicity, we distinguish again the three cases where the only non-vanishing parameter is $\gamma \neq 0$, $\beta \neq 0$ or $\alpha \neq 0$.

When $\gamma \neq 0$, the equation for $\delta \phi$ is given by,

$$
\delta \phi^{\prime \prime}+\frac{r_{\mathrm{s}}\left(r-r_{\mathrm{s}}\right)+2 i\left(r_{\mathrm{s}} r^{5}\right)^{1 / 2}}{r r_{\mathrm{s}}} \delta \phi^{\prime}-\frac{\omega\left[5 i\left(r r_{\mathrm{s}}\right)^{3 / 2}-3 i\left(r^{5} r_{\mathrm{s}}\right)^{1 / 2}+2 \omega r^{4}\right]}{2 r r_{\mathrm{s}}\left(r-r_{\mathrm{s}}\right)^{2}} \delta \phi=0 .
$$

We introduce a new field $\varphi$ defined by

$$
\delta \phi=\kappa(r) \varphi(r)
$$

where $\kappa(r)$ is chosen to eliminate the first-order derivative in the differential equation. This can be achieved with

$$
\kappa(r)=\exp \left[-2 i \omega \sqrt{r / r_{\mathrm{s}}}\left(r+3 r_{\mathrm{s}}\right)\right]\left(\frac{\sqrt{r / r_{\mathrm{s}}}+1}{\sqrt{r / r_{\mathrm{s}}}-1}\right)^{i \omega r_{\mathrm{s}}},
$$

and then $\varphi$ is solution of the second order equation

$$
4 r^{2} \varphi^{\prime \prime}+\varphi=0
$$

which can be solved immediately to get

$$
\varphi(r)=a_{1} \sqrt{r}+a_{2} \sqrt{r} \ln r,
$$

where $a_{1}$ and $a_{2}$ are integration constants. We also notice that we recover the asymptotic behaviours of the scalar mode obtained in (5.25) and (5.26).

The case where $\beta \neq 0$ is treated in exactly the same way. Taking now

$$
\kappa(r)=\exp \left[-2 i \omega \sqrt{r r_{\mathrm{s}}}\right]\left(\frac{\sqrt{r / r_{\mathrm{s}}}+1}{\sqrt{r / r_{\mathrm{s}}}-1}\right)^{i \omega r_{\mathrm{s}}},
$$

we show that the field $\varphi$ satisfies the equation

$$
4 r^{2} \varphi^{\prime \prime}+(4 \lambda+1) \varphi=0,
$$

which, again, can be solved immediately

$$
\varphi(r)=\sqrt{r}\left(a_{+} r^{i \sqrt{\lambda}}+a_{-} r^{-i \sqrt{\lambda}}\right),
$$

where $a_{ \pm}$are constants. We find again that the perturbation is not propagating. Furthermore, these results agree with the full asymptotic analysis of the solutions of the polar system. 
Finally, in the case $\alpha \neq 0$, the equation satisfied by $\delta \phi$ at linear order disappears, since the quadratic Lagrangian for $\delta \phi$ is a total derivative.

[1] K. D. Kokkotas and B. G. Schmidt, "Quasinormal modes of stars and black holes," Living Rev. Rel. 2 (1999) $2, \mathrm{gr}-\mathrm{qc} / 9909058$

[2] H.-P. Nollert, "Topical Review: Quasinormal modes: the characteristic 'sound' of black holes and neutron stars," Class. Quant. Grav. 16 (1999) R159-R216.

[3] E. Berti, V. Cardoso, and A. O. Starinets, "Quasinormal modes of black holes and black branes," Class. Quant. Grav. 26 (2009) 163001, 0905.2975

[4] R. A. Konoplya and A. Zhidenko, "Quasinormal modes of black holes: From astrophysics to string theory," Rev. Mod. Phys. 83 (2011) 793-836, 1102.4014.

[5] E. Berti, V. Cardoso, and C. M. Will, "On gravitational-wave spectroscopy of massive black holes with the space interferometer LISA," Phys. Rev. D 73 (2006) 064030, gr-qc/0512160.

[6] E. Berti, K. Yagi, H. Yang, and N. Yunes, "Extreme Gravity Tests with Gravitational Waves from Compact Binary Coalescences: (II) Ringdown," Gen. Rel. Grav. 50 (2018), no. 5 49, 1801.03587.

[7] D. Langlois and K. Noui, "Degenerate higher derivative theories beyond Horndeski: evading the Ostrogradski instability," JCAP 1602 (2016), no. 02 034, 1510.06930.

[8] J. Ben Achour, M. Crisostomi, K. Koyama, D. Langlois, K. Noui, and G. Tasinato, "Degenerate higher order scalar-tensor theories beyond Horndeski up to cubic order," JHEP 12 (2016) 100, 1608.08135.

[9] D. Langlois, "Dark energy and modified gravity in degenerate higher-order scalar?tensor (DHOST) theories: A review," Int. J. Mod. Phys. D28 (2019), no. 05 1942006, 1811.06271.

[10] Y. Fujii and K. Maeda, The scalar-tensor theory of gravitation. Cambridge Monographs on Mathematical Physics. Cambridge University Press, 7, 2007.

[11] G. W. Horndeski, "Second-order scalar-tensor field equations in a four-dimensional space," Int.J.Theor.Phys. 10 (1974) 363-384.

[12] M. Zumalacárregui and J. García-Bellido, "Transforming gravity: from derivative couplings to matter to second-order scalar-tensor theories beyond the Horndeski Lagrangian," Phys. Rev. D 89 (2014) $064046,1308.4685$.

[13] J. Gleyzes, D. Langlois, F. Piazza, and F. Vernizzi, "Healthy theories beyond Horndeski," Phys. Rev. Lett. 114 (2015), no. 21 211101, 1404.6495.

[14] J. Ben Achour and H. Liu, "Hairy Schwarzschild-(A)dS black hole solutions in degenerate higher order scalar-tensor theories beyond shift symmetry," Phys. Rev. D 99 (2019), no. 6 064042, 1811.05369.

[15] H. Motohashi and M. Minamitsuji, "Exact black hole solutions in shift-symmetric quadratic degenerate higher-order scalar-tensor theories," Phys. Rev. D99 (2019), no. 6 064040, 1901.04658

[16] C. Charmousis, M. Crisostomi, R. Gregory, and N. Stergioulas, "Rotating Black Holes in Higher Order Gravity," Phys. Rev. D 100 (2019), no. 8 084020, 1903.05519.

[17] M. Minamitsuji and J. Edholm, "Black hole solutions in shift-symmetric degenerate higher-order scalar-tensor theories," Phys. Rev. D100 (2019), no. 4 044053, 1907.02072

[18] J. Ben Achour, H. Liu, and S. Mukohyama, "Hairy black holes in DHOST theories: Exploring disformal transformation as a solution-generating method," JCAP 02 (2020) 023, 1910.11017.

[19] M. Minamitsuji and J. Edholm, "Black holes with a nonconstant kinetic term in degenerate higher-order scalar tensor theories," Phys. Rev. D101 (2020), no. 4 044034, 1912.01744

[20] T. Anson, E. Babichev, C. Charmousis, and M. Hassaine, "Disforming the Kerr metric," JHEP 01 (2021) 018, 2006.06461.

[21] J. Ben Achour, H. Liu, H. Motohashi, S. Mukohyama, and K. Noui, "On Rotating Black Holes in DHOST Theories," 2006.07245.

[22] K. Takahashi and H. Motohashi, "General Relativity solutions with stealth scalar hair in quadratic higher-order scalar-tensor theories," JCAP 06 (2020) 034, 2004.03883.

[23] E. Babichev, C. Charmousis, A. Cisterna, and M. Hassaine, "Regular black holes via the Kerr-Schild construction in DHOST theories," JCAP 06 (2020) 049, 2004.00597.

[24] O. Baake, M. F. Bravo Gaete, and M. Hassaine, "Spinning black holes for generalized scalar tensor 
theories in three dimensions," Phys. Rev. D 102 (2020), no. 2 024088, 2005.10869.

[25] E. Babichev, C. Charmousis, and A. Lehbel, "Black holes and stars in Horndeski theory," Class. Quant. Grav. 33 (2016), no. 15 154002, 1604.06402.

[26] E. Babichev, C. Charmousis, and A. Lehbel, "Asymptotically flat black holes in Horndeski theory and beyond," JCAP 1704 (2017) 027, 1702.01938.

[27] M. Minamitsuji and H. Motohashi, "Stealth Schwarzschild solution in shift symmetry breaking theories," Phys. Rev. D98 (2018), no. 8 084027, 1809.06611.

[28] C. de Rham and J. Zhang, "Perturbations of stealth black holes in degenerate higher-order scalar-tensor theories," Phys. Rev. D 100 (2019), no. 12 124023, 1907.00699.

[29] J. Khoury, M. Trodden, and S. S. C. Wong, "Existence and Instability of Novel Hairy Black Holes in Shift-symmetric Horndeski Theories," 2007.01320.

[30] H. Motohashi and S. Mukohyama, "Weakly-coupled stealth solution in scordatura degenerate theory," JCAP 01 (2020) 030, 1912.00378.

[31] T. Kobayashi, H. Motohashi, and T. Suyama, "Black hole perturbation in the most general scalar-tensor theory with second-order field equations I: the odd-parity sector," Phys. Rev. D85 (2012) 084025, 1202.4893. [Erratum: Phys. Rev.D96,no.10,109903(2017)].

[32] T. Kobayashi, H. Motohashi, and T. Suyama, "Black hole perturbation in the most general scalar-tensor theory with second-order field equations II: the even-parity sector," Phys. Rev. D89 (2014), no. $8084042,1402.6740$.

[33] H. Ogawa, T. Kobayashi, and T. Suyama, "Instability of hairy black holes in shift-symmetric Horndeski theories," Phys. Rev. D 93 (2016), no. 6 064078, 1510.07400.

[34] K. Takahashi and T. Suyama, "Linear perturbation analysis of hairy black holes in shift-symmetric Horndeski theories: Odd-parity perturbations," Phys. Rev. D 95 (2017), no. 2 024034, 1610.00432.

[35] E. Babichev, C. Charmousis, G. Esposito-Farèse, and A. Lehébel, "Hamiltonian unboundedness vs stability with an application to Horndeski theory," Phys. Rev. D 98 (2018), no. 10104050 , 1803.11444.

[36] K. Takahashi, H. Motohashi, and M. Minamitsuji, "Linear stability analysis of hairy black holes in quadratic degenerate higher-order scalar-tensor theories: Odd-parity perturbations," Phys. Rev. D100 (2019), no. 2 024041, 1904.03554.

[37] K. Tomikawa and T. Kobayashi, "Perturbations and quasi-normal modes of black holes with time-dependent scalar hair in shift-symmetric scalar-tensor theories," 2101.03790.

[38] K. Takahashi and H. Motohashi, "Black hole perturbations in DHOST theories: Master variables, gradient instability, and strong coupling," JCAP 08 (2021) 013, 2106.07128.

[39] C. Charmousis, M. Crisostomi, D. Langlois, and K. Noui, "Perturbations of a rotating black hole in DHOST theories," Class. Quant. Grav. 36 (2019), no. 23 235008, 1907.02924.

[40] O. J. Tattersall, P. G. Ferreira, and M. Lagos, "General theories of linear gravitational perturbations to a Schwarzschild Black Hole," Phys. Rev. D97 (2018), no. 4 044021, 1711.01992.

[41] R. McManus, E. Berti, C. F. B. Macedo, M. Kimura, A. Maselli, and V. Cardoso, "Parametrized black hole quasinormal ringdown. II. Coupled equations and quadratic corrections for nonrotating black holes," Phys. Rev. D 100 (2019), no. 4 044061, 1906.05155.

[42] D. Langlois, K. Noui, and H. Roussille, "Asymptotics of linear differential systems and application to quasi-normal modes of nonrotating black holes," 2103.14744.

[43] J. Ben Achour, D. Langlois, and K. Noui, "Degenerate higher order scalar-tensor theories beyond Horndeski and disformal transformations," Phys. Rev. D 93 (2016), no. 12 124005, 1602.08398.

[44] M. Crisostomi, K. Koyama, and G. Tasinato, "Extended Scalar-Tensor Theories of Gravity," JCAP 1604 (2016), no. $04044,1602.03119$

[45] A. H. Chamseddine and V. Mukhanov, "Mimetic Dark Matter," JHEP 11 (2013) 135, 1308.5410.

[46] N. Deruelle and J. Rua, "Disformal Transformations, Veiled General Relativity and Mimetic Gravity," JCAP 09 (2014) 002, 1407.0825

[47] K. Takahashi and T. Kobayashi, "Extended mimetic gravity: Hamiltonian analysis and gradient instabilities," JCAP 11 (2017) 038, 1708.02951

[48] D. Langlois, M. Mancarella, K. Noui, and F. Vernizzi, "Mimetic gravity as DHOST theories," JCAP 02 (2019) 036, 1802.03394

[49] D. Langlois, K. Noui, and H. Roussille, "Quadratic DHOST theories revisited," 2012.10218.

[50] D. Langlois and K. Noui, "Hamiltonian analysis of higher derivative scalar-tensor theories," JCAP 
1607 (2016), no. $07016,1512.06820$

[51] D. Langlois, M. Mancarella, K. Noui, and F. Vernizzi, "Effective Description of Higher-Order Scalar-Tensor Theories," JCAP 1705 (2017), no. 05 033, 1703.03797.

[52] E. Babichev and C. Charmousis, "Dressing a black hole with a time-dependent Galileon," JHEP 08 (2014) 106, 1312.3204.

[53] A. Lehbel, E. Babichev, and C. Charmousis, "A no-hair theorem for stars in Horndeski theories," JCAP 1707 (2017) 037, 1706.04989.

[54] E. Ayon-Beato, C. Martinez, and J. Zanelli, "Stealth scalar field overflying a (2+1) black hole," Gen. Rel. Grav. 38 (2006) 145-152, hep-th/0403228.

[55] S. Mukohyama, "Black holes in the ghost condensate," Phys. Rev. D 71 (2005) 104019, hep-th/0502189.

[56] N. Arkani-Hamed, H.-C. Cheng, M. A. Luty, and S. Mukohyama, "Ghost condensation and a consistent infrared modification of gravity," JHEP 05 (2004) 074, hep-th/0312099.

[57] K. Van Aelst, E. Gourgoulhon, P. Grandclément, and C. Charmousis, "Hairy rotating black holes in cubic Galileon theory," Class. Quant. Grav. 37 (2020), no. 3 035007, 1910.08451.

[58] T. Regge and J. A. Wheeler, "Stability of a Schwarzschild singularity," Phys. Rev. 108 (1957) 1063-1069.

[59] A. Ganguly, R. Gannouji, M. Gonzalez-Espinoza, and C. Pizarro-Moya, "Black hole stability under odd-parity perturbations in Horndeski gravity," Class. Quant. Grav. 35 (2018), no. 14 145008, 1710.07669 .

[60] O. J. Tattersall, "Quasi-Normal Modes of Hairy Scalar Tensor Black Holes: Odd Parity," Class. Quant. Grav. 37 (2020), no. 11 115007, 1911.07593.

[61] D. Langlois, R. Saito, D. Yamauchi, and K. Noui, "Scalar-tensor theories and modified gravity in the wake of GW170817," Phys. Rev. D97 (2018), no. 6 061501, 1711.07403.

[62] O. J. Tattersall and P. G. Ferreira, "Quasinormal modes of black holes in Horndeski gravity," Phys. Rev. D97 (2018), no. 10 104047, 1804.08950.

[63] W. Wasow, Asymptotic Expansions for Ordinary Differential Equations. Courier Dover publications, 1965.

[64] W. Balser, "Computation of formal fundamental solutions," Linear Algebra and its Applications 288 (Feb., 1999) 293-312.

[65] M. Barkatou and E. Pflügel, "An Algorithm Computing the Regular Formal Solutions of a System of Linear Differential Equations," Journal of Symbolic Computation 28 (Oct., 1999) 569-587.

[66] E. Pflügel, "A Root-Free Splitting-Lemma for Systems of Linear Differential Equations," arXiv:1911.05837 [cs] (Nov., 2019). arXiv: 1911.05837.

[67] J. L. Blázquez-Salcedo, C. F. B. Macedo, V. Cardoso, V. Ferrari, L. Gualtieri, F. S. Khoo, J. Kunz, and P. Pani, "Perturbed black holes in Einstein-dilaton-Gauss-Bonnet gravity: Stability, ringdown, and gravitational-wave emission," Phys. Rev. D 94 (2016), no. 10 104024, 1609.01286.

[68] J. L. Blázquez-Salcedo, F. S. Khoo, and J. Kunz, "Quasinormal modes of Einstein-Gauss-Bonnet-dilaton black holes," Phys. Rev. D 96 (2017), no. 6 064008, 1706.03262

[69] J. L. Blázquez-Salcedo, D. D. Doneva, S. Kahlen, J. Kunz, P. Nedkova, and S. S. Yazadjiev, "Polar quasinormal modes of the scalarized Einstein-Gauss-Bonnet black holes," Phys. Rev. D 102 (2020), no. 2 024086, 2006.06006.

[70] J. L. Blázquez-Salcedo, D. D. Doneva, S. Kahlen, J. Kunz, P. Nedkova, and S. S. Yazadjiev, "Axial perturbations of the scalarized Einstein-Gauss-Bonnet black holes," Phys. Rev. D 101 (2020), no. 10 104006, 2003.02862.

[71] C. Molina, P. Pani, V. Cardoso, and L. Gualtieri, "Gravitational signature of Schwarzschild black holes in dynamical Chern-Simons gravity," Phys. Rev. D 81 (2010) 124021, 1004.4007.

[72] A. Jansen, "Overdamped modes in Schwarzschild-de Sitter and a Mathematica package for the numerical computation of quasinormal modes," Eur. Phys. J. Plus 132 (2017), no. 12 546, 1709.09178 . 